\title{
QQ-system and non-linear integral equations for scattering amplitudes at strong coupling
}

\author{
Davide Fioravanti, ${ }^{a}$ Marco Rossi $^{b}$ and Hongfei Shu ${ }^{c}$ \\ ${ }^{a}$ Sezione INFN di Bologna, Dipartimento di Fisica e Astronomia, Università di Bologna, \\ Via Irnerio 46, Bologna 40126, Italy \\ ${ }^{b}$ Dipartimento di Fisica dell'Università della Calabria and INFN, Gruppo collegato di Cosenza, \\ Arcavacata di Rende, Cosenza 87036, Italy \\ ${ }^{c}$ Nordita, KTH Royal Institute of Technology and Stockholm University, \\ Roslagstullsbacken 23, Stockholm SE-106 91, Sweden \\ E-mail: fioravanti@bo.infn.it, rossi@cs.infn.it, hongfei.shu@su.se
}

ABSTRACT: We provide the two fundamental sets of functional relations which describe the strong coupling limit in $A d S_{3}$ of scattering amplitudes in $\mathcal{N}=4$ SYM dual to Wilson loops (possibly extended by a non-zero twist $l$ ): the basic $Q Q$-system and the derived $T Q$-system. We use the $T Q$ relations and the knowledge of the main properties of the $Q$-function (eigenvalue of some $Q$-operator) to write the Bethe Ansatz equations, viz. a set of ('complex') non-linear-integral equations, whose solutions give exact values to the strong coupling amplitudes/Wilson loops. Moreover, they have some advantages with respect to the ('real') non-linear-integral equations of Thermodynamic Bethe Ansatz and still reproduce, both analytically and numerically, the findings coming from the latter. In any case, these new functional and integral equations give a larger perspective on the topic also applicable to the realm of $\mathcal{N}=2 \mathrm{SYM}$ BPS spectra.

KEywords: AdS-CFT Correspondence, Bethe Ansatz, Integrable Field Theories

ARXIV EPRINT: 2004.10722 


\section{Contents}

1 Some background and plan of the paper 1

2 Functional relations 3

$\begin{array}{llr}3 & \text { Non-linear integral equations } & 6\end{array}$

4 Analytic computations $\quad 12$

5 Numerical computations $\quad 13$

6 Conformal limit $\quad 16$

$\begin{array}{lll}7 & \text { Conclusions } & 19\end{array}$

\section{Some background and plan of the paper}

Maximally supersymmetric $\mathcal{N}=4$ gauge theory in $4 \mathrm{D}$ has been revealing many integrable features, mainly in the planar limit, which also involve its strong coupling dual string theory [1-3]: for instance, integrable spin-chain hamiltonians and $S$-matrices appear in the computation of anomalous dimensions of composite operators (cf. e.g. [4] and [5-7] for the related Thermodynamic Bethe Ansatz to be compared with the following). Furthermore, a gluon scattering amplitude equals its dual, namely the null polygonal Wilson loop (WL) and the latter admits an expression as quantum string partition function [8]. In particular, at strong coupling, the amplitude is dominated by the classical contribution, i.e. the minimal area of the surface in $A d S_{5}$ ending on the WL in the (boundary) 4D Minkowski.

Now, another integrability issue enters the stage and it will be our main concern in this paper. Albeit our investigation is applicable to the full $A d S_{5}$ space, we shall confine our analysis to the easier $A d S_{3}$ subspace and its classical string solutions. In fact, the problem of finding global coordinates parametrising $A d S_{3}$ is equivalent to solving the two spectral linear problems $D \Psi(z, \bar{z} ; \theta)=0, \bar{D} \Psi(z, \bar{z} ; \theta)=0$, with $z, \bar{z}$ world-sheet coordinates, $\theta$ (complex) spectral parameter and $D, \bar{D}$ linear differential operators depending on a 'potential' $\eta(z, \bar{z})$ (explicitly given by formulæ (2.2), (2.3) below). The latter must satisfy the (Lax) compatibility condition (denoting classical integrability), which in this case is a modified Sinh-Gordon equation - (2.5) below - depending uniquely on an entire function $p$ and its complex conjugate, $\bar{p}$. We choose $p$ to be a polynomial of degree $2 N$ (integer) so that we describe light-like WLs with $4 N+4$ cusps $(4 N-2$ real cross ratios out of these points: the number of real parameters in $p(z)$ if the highest coefficient is 1 and the sum of its zeroes is null) or a scattering amplitude among $4 N+4$ gluons. Besides the aforementioned classical 
integrability, the two coupled linear problems show remarkable quantum integrability properties: in a nutshell Wronskians of solutions are $Q$ and $T$ functions (eigenvalues of some Baxter Q-operator and transfer matrix respectively) of a generalised Baxter setup with moduli (e.g. the complex coefficients of the polynomial $p(z)$ ). This is clearly an extension of the so-called ODE/IM correspondence [9-13], which still maintains the $Q$-functions and their functional relations, the $Q Q$-systems, as fundamental building blocks.

Actually, some $T$ s and $Y$ s are already present in previous papers $[14],{ }^{1}$ while here and in the forthcoming paper [16] we make the construction more general and extend by founding it on the basic $Q$ functions and their $Q Q$ relations. Next, we derive the fundamental generalised Baxter $T Q$ relations and easily from these the Bethe Ansatz equations which are not so tractable because of their infinite number. Therefore, we convert them into a system of $2 N$ non-linear integral equations (NLIEs) depending on the $2 N$-ple of the coefficients of the polynomial $p(z)$. Crucially, for the whole construction of our theory we make use of two distinct discrete symmetries, $\hat{\Omega}$ and $\hat{\Pi}$, of the linear associated problems $D \Psi=0$, $\bar{D} \Psi=0$, which, yet, gain their effectiveness by a non-trivial action on the vacuum $\Psi$ (not invariant): in other words these are broken symmetries giving birth to the characteristic quantities of integrability ( $Q \mathrm{~s}, T \mathrm{~s}$ and $Y \mathrm{~s}$, which are generically the generators of the (local and non-local) conserved charges). Eventually, we propose an expression for the free energy in terms of solutions of the NLIEs. We claim and verify both analytically and numerically in many cases that our expression coincides with the free energy of [14] written in terms of solutions of Thermodynamic Bethe Ansatz (TBA) equations.

In fact, the NLIEs show many advantages with respect to TBA, at least for the polynomials we studied, which, technically speaking, are in the so-called maximal chamber [14]: for polynomials of degree $2 N$ their number is $2 N$, whilst the number of TBA equations is $2 N(2 N-1) / 2$ (quadratic grow); moreover, the procedure to write NLIEs is undoubtedly simpler than (and should shed light on) the corresponding one for TBA, which passes through $(N-1)(2 N-1)$ 'wall crossings'. In addition, from the ideological point of view the NLIEs, being equivalent to the infinite Bethe Ansatz equations, lie at the heart of the integrability of the problem, and one can express in terms of their solutions all the relevant quantities, such as the $Q$ functions, the transfer matrices eigenvalues $T$ and the $Y$ functions. We will provide explicit expressions for all that at the end of section 3.

The paper is organised as follows: in the next section 2 we sketch quickly the derivation of all the functional relations which will be better analysed in [16]. Then, section 3 is the core of the paper, as we derive a system of NLIEs and propose an expression for the free energy in terms of solutions of the NLIEs. Our results for the free energy are tested analytically in some simple cases (section 4) and numerically in many examples (section 5), finding perfect agreement with analogous computations made by using TBA. Finally in section 6 we study the 'conformal limit' in which the auxiliary linear problem reduces to a Schrödinger equation: our set of NLIEs reduces - as expected - to the NLIEs introduced in $[17,18]$.

\footnotetext{
${ }^{1}$ We also suggest to consult also [15] for the first appearance of TBA from the same differential problem (Hitchin or SG systems), but for the very important problem of BPS spectra and wall-crossing in $\mathcal{N}=$ 2 SYM.
} 


\section{Functional relations}

As sketched in the introduction, we start from the linear problem (the bar means complex conjugation)

$$
D \Psi=0, \quad \bar{D} \Psi=0,
$$

with $^{2}$

$$
\begin{aligned}
& D=\partial_{z}+\frac{1}{2} \partial_{z} \eta \sigma^{3}-e^{\theta}\left[\sigma^{+} e^{\eta}+\sigma^{-} p(z, \vec{c}) e^{-\eta}\right] \\
& \bar{D}=\partial_{\bar{z}}-\frac{1}{2} \partial_{\bar{z}} \eta \sigma^{3}-e^{-\theta}\left[\sigma^{-} e^{\eta}+\sigma^{+} \bar{p}(z, \vec{c}) e^{-\eta}\right]
\end{aligned}
$$

The polynomial

$$
p(z, \vec{c})=z^{2 N}+\sum_{n=0}^{2 N-2} c_{n} z^{n}
$$

depends on a $2 N-1$-ple of complex coefficients $\vec{c}=\left(c_{0}, \ldots, c_{2 N-2}\right)$ or moduli.

Zero curvature condition $[D, \bar{D}]=0$ constraints the 'potential' $\eta(z, \bar{z} ; \vec{c})$ to be solution of the classical modified Sinh-Gordon equation

$$
\partial_{z} \partial_{\bar{z}} \eta-e^{2 \eta}+p(z, \vec{c}) \bar{p}(z, \vec{c}) e^{-2 \eta}=0 .
$$

To completely specify $\eta$, we impose the condition $\eta=l \ln z \bar{z}+O(1)$ as $|z| \rightarrow 0$, for $|l|<1 / 2$. Operating in such a way we are extending framework of [20] by introducing the moduli $\vec{c}$ (besides $c_{0}$ ) and the $A d S_{3}$ string problem representing the WL [21] which is regular in $z=0$, i.e. $l=0$, with a twist $l .^{3}$ The same extension holds for the series of homogeneous Sine-Gordon models [23, 24].

The linear problem has two symmetries. The first one acts as

$$
\hat{\Omega}: \quad z \rightarrow z e^{\frac{i \pi}{N}}, \quad \theta \rightarrow \theta-\frac{i \pi}{N}, \quad \vec{c} \rightarrow \vec{c}^{R}, \quad \vec{c}^{R}=\left(c_{0}, c_{1} e^{-\frac{i \pi}{N}}, \ldots, c_{n} e^{-\frac{i \pi n}{N}}, \ldots, c_{2 N-2} e^{\frac{2 i \pi}{N}}\right) .
$$

The $\hat{\Omega}$ symmetry leaves invariant the polynomial $p$ and the function $\eta: p\left(z e^{\frac{i \pi}{N}}, \vec{c}^{R}\right)=$ $p(z, \vec{c}), \eta(z, \bar{z} ; \vec{c})=\eta\left(z e^{\frac{i \pi}{N}}, \bar{z} e^{-\frac{i \pi}{N}} ; \vec{c}^{R}\right)$. However, it produces a change of sheet and then $\sqrt{p\left(z e^{\frac{i \pi}{N}}, \vec{c}^{R}\right)}=-\sqrt{p(z, \vec{c})}$. This symmetry involving rotations of the moduli appeared in the context of a Schrödinger equation with polynomial potential in [25] and, recently, in $[26,27]$ : we will analyse this as limiting 2D conformal case of our massive one in section 6 . Another symmetry of the linear operators $D$ and $\bar{D}$ is given by the transformation

$$
\hat{\Pi}: D \rightarrow \sigma^{3} D \sigma^{3}, \quad \bar{D} \rightarrow \sigma^{3} \bar{D} \sigma^{3}, \quad \theta \rightarrow \theta-i \pi .
$$

\footnotetext{
${ }^{2}$ We are considering $s u(2)$ fundamental representations: $\sigma_{i}$ are Pauli matrices and $\sigma^{ \pm}=\frac{1}{2}\left(\sigma_{1} \pm i \sigma_{2}\right)$; for generalisations see for instance [19].

${ }^{3}$ When $l(l+1)$ is nonzero, the solution of the linear problem will have a non-trivial monodromy. Mapping to the classical solution of the worldsheet, one finds a periodic Wilson line, which is dual to a form factor in the context of scattering amplitude/Wilson loop duality. A similar situation has been studied in [22].
} 
Now, following [20], we can fix two couples of solutions. The first one, $\Psi_{ \pm}(z ; \theta, \vec{c})$, is completely specified by the expansion around $z=\rho e^{i \varphi}=0, \bar{z}=\rho e^{-i \varphi}=0$

$$
\Psi_{+} \simeq \frac{1}{\sqrt{\cos \pi l}}\left(\begin{array}{c}
0 \\
e^{(i \varphi+\theta) l}
\end{array}\right), \quad \Psi_{-} \simeq \frac{1}{\sqrt{\cos \pi l}}\left(\begin{array}{c}
e^{-(i \varphi+\theta) l} \\
0
\end{array}\right),|z| \rightarrow 0 .
$$

The second couple (a Jost basis) is given by the solutions $\Xi(z ; \theta, \vec{c})$ and $\Xi_{1}(z ; \theta, \vec{c})$ with following asymptotic leading expansion around $z=+\infty$

$$
\begin{aligned}
& \Xi(z ; \theta, \vec{c}) \simeq\left(\begin{array}{c}
e^{-\frac{i N \varphi}{2}} \\
-e^{\frac{i N \varphi}{2}}
\end{array}\right) \exp \left[-e^{\theta} \int^{z} d z \sqrt{p(z, \vec{c})}-e^{-\theta} \int^{\bar{z}} d \bar{z} \sqrt{\bar{p}(z, \vec{c})}\right] \\
& \Xi_{1}(z ; \theta, \vec{c}) \simeq-i e^{i \Phi(\theta, \vec{c})} \sigma^{3}\left(\begin{array}{c}
e^{-\frac{i N \varphi}{2}} \\
-e^{\frac{i N \varphi}{2}}
\end{array}\right) \exp \left[e^{\theta} \int^{z} d z \sqrt{p(z, \vec{c})}+e^{-\theta} \int^{\bar{z}} d \bar{z} \sqrt{\bar{p}(z, \vec{c})}\right] .
\end{aligned}
$$

We found convenient to modify the second solution by introducing the phase

$$
\Phi(\theta, \vec{c})=\frac{\pi}{N} B_{-1}(\vec{c}) e^{\theta}-\frac{\pi}{N} \bar{B}_{-1}(\vec{c}) e^{-\theta},
$$

where the coefficient $B_{-1}(\vec{c})$ appears in the large $|z|$ expansion of $\sqrt{p(z, \vec{c})}$ :

$$
\sqrt{p(z, \vec{c})}=z^{N}+\cdots+B_{-1}(\vec{c}) z^{-1}+O\left(z^{-2}\right) .
$$

This means that the function $\Phi(\theta, \vec{c})=0$ is non zero only if $N$ is integer. Important properties of $\Phi$ are

$$
\Phi(\theta, \vec{c})=-\Phi\left(\theta-\frac{i \pi}{N}, \vec{c}^{R}\right)=-\Phi(\theta+i \pi, \vec{c})
$$

the first of which descends from $B_{-1}(\vec{c})=-e^{-\frac{i \pi}{N}} B_{-1}\left(\vec{c}^{R}\right)$.

These sets of solutions transforms in a nice way under the symmetries $\hat{\Omega}$ and $\hat{\Pi}$ :

$$
\begin{aligned}
\hat{\Omega} \Psi_{ \pm}(z ; \theta, \vec{c}) & =\Psi_{ \pm}\left(z e^{\frac{i \pi}{N}} ; \theta-\frac{i \pi}{N}, \vec{c}^{R}\right)=\Psi_{ \pm}(z ; \theta, \vec{c}) \\
\hat{\Pi} \Psi_{ \pm}(z ; \theta, \vec{c}) & =\Psi_{ \pm}(z ; \theta-i \pi, \vec{c})=\mp e^{\mp i \pi l} \sigma^{3} \Psi_{ \pm}(z ; \theta, \vec{c}) \\
\hat{\Omega} \Xi(z ; \theta, \vec{c}) & =\Xi\left(z e^{\frac{i \pi}{N}} ; \theta-\frac{i \pi}{N}, \vec{c}^{R}\right)=\Xi_{1}(z ; \theta, \vec{c}) \\
(\hat{\Omega} \circ \hat{\Pi}) \Xi(z ; \theta, \vec{c}) & =\Xi\left(z e^{\frac{i \pi}{N}} ; \theta-i \pi-\frac{i \pi}{N}, \vec{c}^{R}\right)=-i \sigma^{3} e^{-i \Phi(\theta, \vec{c})} \Xi(z ; \theta, \vec{c}) .
\end{aligned}
$$

Now, it is natural to connect the two sets of solutions by means of $z$ independent coefficients $Q_{ \pm}(\theta, \vec{c})$ :

$$
\Xi(z ; \theta, \vec{c})=Q_{+}(\theta, \vec{c}) \Psi_{-}(z ; \theta, \vec{c})+Q_{-}(\theta, \vec{c}) \Psi_{+}(z ; \theta, \vec{c}) .
$$

Then, applying $\hat{\Omega}$ to both sides of this equation

$$
\Xi_{1}(z ; \theta, \vec{c})=Q_{+}\left(\theta-\frac{i \pi}{N}, \vec{c}^{R}\right) \Psi_{-}(z ; \theta, \vec{c})+Q_{-}\left(\theta-\frac{i \pi}{N}, \vec{c}^{R}\right) \Psi_{+}(z ; \theta, \vec{c}),
$$


taking the determinant of both equations and using the explicit results $\operatorname{det}\left(\Xi, \Xi_{1}\right)=$ $-2 i e^{i \Phi(\theta, \vec{c})}$ and $\operatorname{det}\left(\Psi_{+}, \Psi_{-}\right)=-1 / \cos \pi l$, we end up with a functional relation that may be considered the foundation of the integrability of the theory, the $Q Q$-system,

$Q_{+}\left(\theta+\frac{i \pi}{2 N}, \vec{c}\right) Q_{-}\left(\theta-\frac{i \pi}{2 N}, \vec{c}^{R}\right)-Q_{+}\left(\theta-\frac{i \pi}{2 N}, \vec{c}^{R}\right) Q_{-}\left(\theta+\frac{i \pi}{2 N}, \vec{c}\right)=-2 i e^{i \Phi\left(\theta+\frac{i \pi}{2 N}, \vec{c}\right)} \cos \pi l$.

This form for the $Q Q$-system is very peculiar since it involves rotations of the moduli and the presence of the extra phase $\Phi$ as well. Because of the presence of different moduli, relations (2.20) are not closed, similarly to functional relations present at the level of $Y$ systems in [28]. More in general, we can think of (2.20) as extensions of analogous relations underlying integrability in the case of $\mathcal{N}=4 \mathrm{SYM}$ in $4 \mathrm{D}$ and $\mathcal{N}=6$ SCS in 3D and which are often called quantum spectral curves $[29,30]$.

Using together $\hat{\Omega}$ and $\hat{\Pi}$ on equation (2.18) we obtain a particular form of quasiperiodicity involving the rotation of the moduli $\vec{c}$

$$
Q_{ \pm}\left(\theta-i \pi-\frac{i \pi}{N}, \vec{c}^{R}\right)=e^{\mp i \pi\left(l+\frac{1}{2}\right)} e^{-i \Phi(\theta, \vec{c})} Q_{ \pm}(\theta, \vec{c}) .
$$

Quasi-periodicity (2.21) allows us to bring the $Q Q$-system (2.20) in a form which does not involve rotations of the moduli and the phase $\Phi$ :

$$
e^{i \pi l} Q_{+}(\theta, \vec{c}) Q_{-}(\theta+i \pi, \vec{c})+e^{-i \pi l} Q_{-}(\theta, \vec{c}) Q_{+}(\theta+i \pi, \vec{c})=-2 \cos \pi l .
$$

Another useful property of $Q_{ \pm}$is its behaviour under complex conjugation

$$
Q_{ \pm}(\theta, \vec{c})=-\bar{Q}_{\mp}(-\bar{\theta}, \vec{c}),
$$

which derives from the relations

$$
\sigma^{1} \bar{\Xi}(z ;-\theta, \vec{c})=-\Xi(z ; \bar{\theta}, \vec{c}), \quad \sigma^{1} \bar{\Psi}_{ \pm}(z ;-\theta, \vec{c})=\Psi_{\mp}(z ; \bar{\theta}, \vec{c}) .
$$

In addition, usual real-analyticity

$$
\bar{Q}_{ \pm}(\theta, \vec{c})=Q_{ \pm}(\bar{\theta}, \overline{\vec{c}})
$$

holds.

In order to write NLIEs we need to introduce the transfer matrix $T$, which we define as a quadratic construct of $Q$ 's:

$$
T(\theta, \vec{c})=\frac{i}{2 \cos \pi l}\left[e^{-2 i \pi l} Q_{+}(\theta+i \pi, \vec{c}) Q_{-}(\theta-i \pi, \vec{c})-e^{2 i \pi l} Q_{+}(\theta-i \pi, \vec{c}) Q_{-}(\theta+i \pi, \vec{c})\right] .
$$

Using the $Q Q$ system, we get the $T Q$-relation or $T Q$-system,

$$
T(\theta, \vec{c}) Q_{ \pm}(\theta ; \vec{c})=e^{ \pm i \pi\left(l+\frac{1}{2}\right)} Q_{ \pm}(\theta-i \pi ; \vec{c})+e^{\mp i \pi\left(l+\frac{1}{2}\right)} Q_{ \pm}(\theta+i \pi ; \vec{c}) .
$$

Importantly, it follows from their construction that both $T(\theta, \vec{c})$ and $Q_{ \pm}(\theta ; \vec{c})$ are analytic functions of $\theta$. This information will be used in the next section to derive in quite a standard way a system of Bethe equations and NLIEs. 
We stress that the construction of $Q Q$ and $T Q$-systems was possible thanks to the use of the $\hat{\Omega}$ symmetry. This symmetry was not used in [14], but we underline its importance to unveil the basics of integrability of the problem. More importantly, the $\hat{\Omega}$ symmetry is essential when differential operators have two irregular singularities, like in Liouville ODE [31].

The last ingredient for the derivation of NLIEs is the leading behaviour of $Q_{ \pm}$when $\operatorname{Re} \theta \rightarrow \pm \infty$. This is obtained by studying the linear problem (2.1) at large $\theta$. Details will be reported in the publication [16]. We give here only the final result:

$$
\ln Q_{ \pm}\left(\theta+i \pi \frac{N+1}{2 N} ; \vec{c}\right) \sim-\left(w_{0}(\vec{c})+\alpha(\vec{c})\right) e^{\theta}-\left(\bar{w}_{0}(\vec{c})+\bar{\alpha}(\vec{c})\right) e^{-\theta},
$$

with

$$
w_{0}(\vec{c})=-\int_{0}^{+\infty} d x\left[\sqrt{P(x, \vec{c})}-q_{N}(x, \vec{c})\right], \quad \alpha(\vec{c})=\frac{\pi}{2 N} B_{-1}(\vec{c}) e^{\frac{i \pi}{2 N}} .
$$

The function $P(x, \vec{c})$ is a polynomial, related to $p(x, \vec{c})=\sum_{n=0}^{2 N} c_{n} x^{n}$ by a change of sign and a half rotation of coefficients $\vec{c}$ :

$$
P(x, \vec{c})=\sum_{n=0}^{2 N} c_{n} e^{i \pi \frac{2 N-n}{2 N}} x^{n} .
$$

The function $q_{N}$ is another polynomial, defined by the condition

$$
\sqrt{P(x, \vec{c})}=q_{N}(x)+o(1 / x), \quad x \rightarrow+\infty,
$$

which assures convergence of the integration in (2.29).

\section{Non-linear integral equations}

By using the $T Q$ system (2.27), the quasiperiodicity property of $Q(2.21)$ and its asymptotic behaviour (2.28), we can write a set of NLIEs. As a preliminary step, we introduce the function $Q(\theta ; k, \vec{c})$ which, when $\operatorname{sgn} k= \pm$, equals $Q_{ \pm}(\theta ; \vec{c})$, with $l= \pm 2 k-\frac{1}{2}$, respectively. Then, we may think of extending (3.24) of [20] by writing $Q(\theta ; k, \vec{c})$ as a product over its zeroes which implements ${ }^{4}$ quasiperiodicity and property (2.23), i.e. $Q(\theta ; k, \vec{c})=-\bar{Q}(-\bar{\theta} ;-k, \vec{c})$ :

$$
\begin{aligned}
Q(\theta ; k, \vec{c})= & e^{\frac{\theta N}{\pi(N+1)} \Phi(\theta, \vec{c})} C(k) e^{\frac{2 \theta N k}{1+N}} \prod_{p=0}^{2 N-1} \prod_{n=0}^{+\infty}\left(1-e^{\frac{\theta}{1+N}} e^{-\frac{i \pi p}{N}} e^{-\frac{\theta_{n}\left(\vec{c}_{p} ; k\right)}{1+N}}\right) \\
& \cdot \prod_{p=0}^{2 N-1}\left(1-e^{-\frac{\theta}{1+N}} e^{\frac{i \pi p}{N}} e^{-\frac{\bar{\theta}_{n}\left(\vec{c}_{p} ;-k\right)}{1+N}}\right)=e^{-i \Phi(\theta, \vec{c})} Q\left(\theta+i \pi+\frac{i \pi}{N} ; k, \vec{c}_{-1}\right) e^{-2 i \pi k}
\end{aligned}
$$

\footnotetext{
${ }^{4}$ The next formula (3.1) is not the only possible with these implementations, but it seems to us the simplest one.
} 
with $C(k)=-C(-k)$ a real constant and $\operatorname{Re} \theta_{n}\left(\vec{c}_{p} ; k\right)$ positive. ${ }^{5}$ In the case of [20], i.e. $c_{n}=0, n=1, \ldots, 2 N-2, \theta_{n}\left(\vec{c}_{p} ; k\right)$ are real and do not depend on $p$ : then, formula (3.1) reduces to (3.24) of [20]. Remembering the form of the $p$ times rotated $2 N-1$-ple

$$
\vec{c}_{p}=\left(c_{0}, c_{1} e^{-\frac{i \pi p}{N}}, \ldots, c_{n} e^{-\frac{i \pi p n}{N}}, \ldots, c_{2 N-2} e^{\frac{2 i \pi p}{N}}\right),
$$

we expect $2 N$ NLIEs, since $2 N$ is the number of discrete rotations (including the identity, i.e. the number of independent sets of $\vec{c}_{p}$ ) of the group acting on the moduli space. In order to write the NLIEs, we recall the $T Q$-system (2.27)

$$
T(\theta, \vec{c}) Q(\theta ; k, \vec{c})=e^{2 i \pi k} Q(\theta-i \pi ; k, \vec{c})+e^{-2 i \pi k} Q(\theta+i \pi ; k, \vec{c}),
$$

which indicates, as usual, that the zeroes of $Q$ are indeed the Bethe roots, $\theta_{n}(\vec{c} ; k)$. This suggests us the definition of their counting function [32, 33]

$$
Z(\theta ; k, \vec{c})=i \ln \left[\frac{Q(\theta-i \pi ; k, \vec{c})}{Q(\theta+i \pi ; k, \vec{c})} e^{4 \pi i k}\right],
$$

so that the Bethe Ansatz equations take the form

$$
e^{i Z\left(\theta_{n}\left(\vec{c}_{p} ; k\right) ; k, \vec{c}_{p}\right)}=-1, e^{i Z\left(-\bar{\theta}_{n}\left(\vec{c}_{p} ;-k\right) ; k, \vec{c}_{p}\right)}=-1, p=0, \ldots, 2 N-1,
$$

and equivalent ones obtained by use of the periodicity of the counting function $Z(\theta ; k, \vec{c})=$ $Z\left(\theta+i \pi+\frac{i \pi}{N} ; k, \vec{c}_{-1}\right)$. Therefore it is very relevant to recast the counting function in a more explicit form as

$$
Z(\theta ; k, \vec{c})=-\frac{4 \pi k+2 N \Phi(\theta, \vec{c})}{1+N}+i \ln \left(\prod_{m=0}^{2 N-1} \prod_{n} \frac{\sinh \left[\frac{i \pi-\theta+\theta_{n}\left(\vec{c}_{m} ; k\right)}{2(1+N)}+\frac{i \pi m}{2 N}\right] \sinh \left[\frac{i \pi-\theta-\bar{\theta}_{n}\left(\vec{c}_{m} ;-k\right)}{2(1+N)}+\frac{i \pi m}{2 N}\right]}{\sinh \left[\frac{\theta+i \pi-\theta_{n}\left(\vec{c}_{m} ; k\right)}{2(1+N)}-\frac{i \pi m}{2 N}\right] \sinh \left[\frac{\theta+i \pi+\bar{\theta}_{n}\left(\vec{c}_{m} ;-k\right)}{2(1+N)}-\frac{i \pi m}{2 N}\right]}\right) .
$$

In order to understand the position of the Bethe roots we refer to the behaviour for large $\theta$ of $Z\left(\theta ; k, \vec{c}_{m}\right)$. We claim that, when $\operatorname{Re} \theta \rightarrow \pm \infty$,

$$
\begin{aligned}
Z\left(\theta ; k, \vec{c}_{m}\right) & \simeq \frac{r\left(\vec{c}_{m}\right)}{2} e^{\theta}-\frac{\bar{r}\left(\vec{c}_{m}\right)}{2} e^{-\theta}=\left|r\left(\vec{c}_{m}\right)\right| \sinh \left(\theta+i \phi_{m}\right) \\
r\left(\vec{c}_{m}\right) & =-2 w_{0}\left(\vec{c}_{m}\right) e^{-\frac{i \pi}{2 N}}-2 w_{0}\left(\vec{c}_{m-1}\right) e^{\frac{i \pi}{2 N}}+\delta r\left(\vec{c}_{m}\right)=r_{0}\left(\vec{c}_{m}\right)+\delta r\left(\vec{c}_{m}\right), \\
\delta r(\vec{c}) & =-\frac{2 \pi}{N} B_{-1}(\vec{c})
\end{aligned}
$$

where we introduced the adimensional quantity $r\left(\vec{c}_{m}\right)=\left|r\left(\vec{c}_{m}\right)\right| e^{i \phi_{m}}$ and used the property $\bar{w}_{0}(\vec{c})=w_{0}\left(\overline{\vec{c}}_{-1}\right)$. Relation (3.7) comes from (3.4), (2.28).

From these definitions it follows that the quantity $r(\vec{c})$ is obtained by an integration on a contour $\Gamma$ defined by $]-i \infty,-i 0] \bigcup[0,+\infty[$ :

$$
r\left(\vec{c}_{1}\right)=2 e^{\frac{i \pi}{2 N}} \int_{\Gamma} d z\left[\sqrt{P(z, \vec{c})}-q_{N}(z, \vec{c})\right] .
$$

\footnotetext{
${ }^{5}$ To simplify notations we called $\vec{c}_{p}$ the $p$ times rotated vector (3.2).
} 


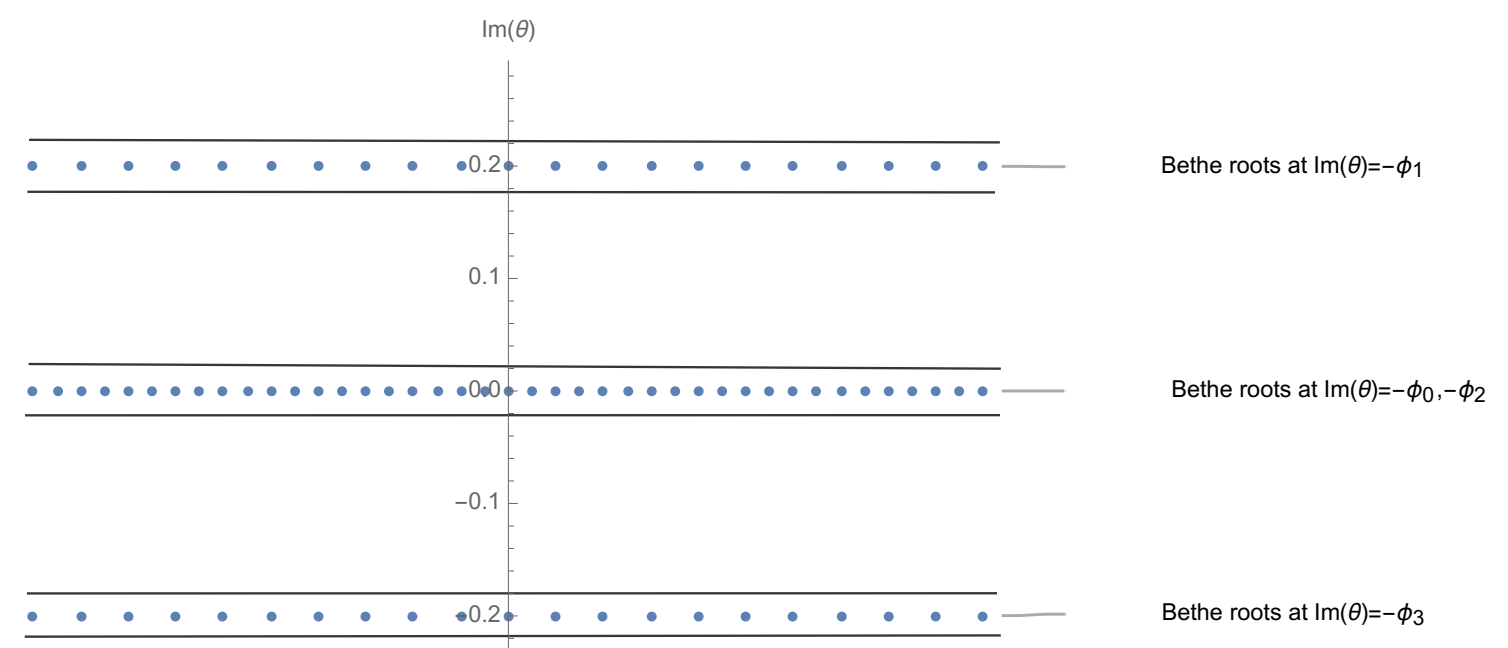

Figure 1. An "ideal" example of the Bethe roots for the polynomial $p(z, \vec{c})=z^{4}+z^{2}-\frac{1}{2} z-1$. The Bethe roots arrange along $\operatorname{Im}(\theta)=-\phi_{0},-\phi_{1},-\phi_{2},-\phi_{3}$. We suppose that for finite $\theta_{n}$ the position of the Bethe roots does not displace much from such lines of blue dots. Summing over Bethe roots is traded into integrating in anticlockwise direction the indicator $\frac{d / d z e^{i Z(z)}}{1+e^{i Z(z)}}$ along a contour (black line) surrounding the roots. After extracting a linear term in $Z$, one gets expression (3.11) below.

In addition, from the asymptotic behaviour (3.7) we deduce that, for very large $\theta_{n}$, $\operatorname{Im} \theta_{n}\left(\vec{c}_{m} ; k\right)=-\operatorname{Im} \bar{\theta}_{n}\left(\vec{c}_{m} ;-k\right)=-\phi_{m}$. We suppose that for finite $\theta_{n}$ the position of the Bethe roots does not displace much from such lines. In figure 1, we provide an "ideal" example of the Bethe roots.

Then, a general sum over the Bethe roots $\theta_{n}\left(\vec{c}_{m} ; k\right),-\bar{\theta}_{n}\left(\vec{c}_{m} ;-k\right)$ can be converted into integrals by using Cauchy theorem [32,33], which fact for $Z$ itself boils down, after standard manipulations, to

$$
\begin{aligned}
Z(\theta ; k, \vec{c})= & -\frac{4 \pi k+2 N \Phi(\theta, \vec{c})}{1+N}+\sum_{m=0}^{2 N-1} \int_{-\infty-i \phi_{m}}^{+\infty-i \phi_{m}} d x K_{m}(\theta-x) Z\left(x ; k, \vec{c}_{m}\right)- \\
& -2 \sum_{m=0}^{2 N-1} \int_{-\infty-i \phi_{m}}^{+\infty-i \phi_{m}} d x\left[K_{m}(\theta-x-i \epsilon) L_{+}\left(x ; k, \vec{c}_{m}\right)-K_{m}(\theta-x+i \epsilon) L_{-}\left(x ; k, \vec{c}_{m}\right)\right],
\end{aligned}
$$

with $\epsilon>0$ and $L_{ \pm}(x ; k, \vec{c})=\frac{1}{2 i} \ln \left[1+e^{ \pm i Z(x \pm i \epsilon ; k, \vec{c})}\right]$. The positive number $\epsilon$ is chosen in such a way that integration contour contains all the Bethe roots, i.e. $\left|\operatorname{Im} \theta_{n}\left(\vec{c}_{m} ; k\right)+\phi_{m}\right|<$ $\epsilon, \forall n, m$.

The kernels $K_{m}(x)$ equal

$$
K_{m}(x)=\frac{1}{2 \pi i} \frac{d}{d x} \ln \left(\frac{\sinh \left[\frac{i \pi+x}{2(1+N)}-\frac{i \pi m}{2 N}\right]}{\sinh \left[\frac{i \pi-x}{2(1+N)}+\frac{i \pi m}{2 N}\right]}\right)=K_{m+2 N}(x),
$$

and in Fourier transform read

$$
\hat{K}_{0}(p)=-\frac{\sinh N \pi p}{\sinh (1+N) \pi p}, \quad \hat{K}_{m}(p)=\frac{\sinh \pi p}{\sinh (1+N) \pi p} e^{(1+N) \pi p\left(\frac{m}{N}-1\right)} \quad \text { for } \quad 1 \leq m \leq 2 N-1 .
$$


Using the periodicity $K_{m}(x)=K_{m+2 N}(x)$ of the kernels and bringing the integrations on the real axis, we can also write

$$
\begin{aligned}
Z\left(\theta-i \phi_{n} ; k, \vec{c}_{n}\right)= & -\frac{4 \pi k+2 N \Phi\left(\theta-i \phi_{n}, \vec{c}_{n}\right)}{1+N} \\
& +\sum_{m=0}^{2 N-1} \int_{-\infty}^{+\infty} d x K_{m-n}\left(\theta-x+i \phi_{m}-i \phi_{n}\right) Z\left(x-i \phi_{m} ; k, \vec{c}_{m}\right)- \\
& -2 \sum_{m=0}^{2 N-1} \int_{-\infty}^{+\infty} d x\left[K_{m-n}\left(\theta-x+i \phi_{m}-i \phi_{n}-i \epsilon\right) L_{+}\left(x-i \phi_{m} ; k, \vec{c}_{m}\right)-\right. \\
& \left.-K_{m-n}\left(\theta-x+i \phi_{m}-i \phi_{n}+i \epsilon\right) L_{-}\left(x-i \phi_{m} ; k, \vec{c}_{m}\right)\right] .
\end{aligned}
$$

Passing to Fourier transforms, we define the matrix $\hat{M}_{i, j}(p)=\delta_{i, j}-e^{p \phi_{i}} \hat{K}_{j-i}(p) e^{-p \phi_{j}}$, whose determinant is explicitly computed:

$$
\operatorname{det} \hat{M}(p)=\prod_{m=0}^{2 N-1}\left(1-\sum_{n=0}^{2 N-1} \hat{K}_{n}(p) e^{\frac{i \pi n m}{N}}\right)=\left(2 \cosh \frac{\pi p}{2}\right)^{2 N} \frac{\sinh \pi p}{\sinh \pi p(1+N)}
$$

The inversion of $\hat{M}_{i, j}(p)$ allows us to write the final set of NLIEs. The driving term for the $n$-th equation equals

$$
-2 \pi k-\Phi\left(\theta-i \phi_{n}, \vec{c}_{n}\right)=-2 \pi k-\frac{\pi}{N}\left(B_{-1}(\vec{c}) e^{i \pi \frac{N+1}{N} n} e^{\theta-i \phi_{n}}-\bar{B}_{-1}(\vec{c}) e^{i \pi \frac{N-1}{N} n} e^{-\theta+i \phi_{n}}\right)
$$

plus zero modes of the matrix $\hat{M}_{i, j}$, i.e. solutions of the homogeneous equation $\hat{M}_{i, j} c_{j}=0$. In this respect, we remark the following property

$$
\left(1-\sum_{n=0}^{2 N-1} \hat{K}_{n}(p) e^{\frac{i \pi n m}{N}}\right) \delta(p \pm i)=0
$$

which holds for all $m=0, \ldots, 2 N-1$, except when $m=N-1$ for $\delta(p-i)$ and for $m=N+1$ for $\delta(p+i)$ (therefore, this two 'problems' appear only for integer $N$ ). This means that zero modes are linear combinations of vectors with the form $c_{n}^{( \pm, m)}=e^{\mp i \phi_{n}} e^{\frac{i \pi n m}{N}} \delta(p \pm i)$, with $m=0, \ldots, 2 N-1$ labeling the independent solutions - with the two exceptions mentioned before. We remark that the quantities $r_{0}\left(\vec{c}_{n}\right) e^{\theta-i \phi_{n}}, \vec{r}_{0}\left(\vec{c}_{n}\right) e^{-\theta+i \phi_{n}}$ can be constructed out of the zero modes, i.e. we have the relation

$$
\sum_{n=0}^{2 N-1} e^{-\frac{i \pi(N+1)}{N} n} r_{0}\left(\vec{c}_{n}\right)=0
$$

which we have numerically verified, see table 3 and table 6 in section 5 . Adding then the last two terms of (3.15) coming from the extra phase $\Phi$, we completely reconstruct the asymptotic behaviour (3.7) previously announced, i.e.

$$
Z\left(\theta-i \phi_{n} ; k, \vec{c}_{n}\right) \sim\left|r\left(\vec{c}_{n}\right)\right| \sinh \theta .
$$


Putting everything together, the final equations are $(0 \leq n \leq 2 N-1)$ :

$$
\begin{aligned}
Z\left(\theta-i \phi_{n} ; k, \vec{c}_{n}\right)= & \left|r\left(\vec{c}_{n}\right)\right| \sinh \theta-2 \pi k \\
& +2 \sum_{m=0}^{2 N-1} \int_{-\infty}^{+\infty} d x\left[G_{m-n}^{(N)}\left(\theta-x-i \phi_{n}+i \phi_{m}-i \epsilon\right) L_{+}\left(x-i \phi_{m} ; k, \vec{c}_{m}\right)-\right. \\
& \left.-G_{m-n}^{(N)}\left(\theta-x-i \phi_{n}+i \phi_{m}+i \epsilon\right) L_{-}\left(x-i \phi_{m} ; k, \vec{c}_{m}\right)\right] .
\end{aligned}
$$

We now give the explicit form for the kernels: the periodicity $G_{m}^{(N)}(x)=G_{m+2 N}^{(N)}(x)$ is understood. For small $N$ one can easily find analytically $(1 \leq m \leq 2 N-1)$ :

$$
\begin{array}{ll}
\hat{G}_{0}^{(1 / 2)}(p)=\frac{1}{4 \cosh ^{2} \frac{\pi p}{2}}, & \hat{G}_{0}^{(1)}(p)=-\hat{G}_{1}^{(1)}(p)=\frac{1}{4 \cosh ^{2} \frac{\pi p}{2}} \\
\hat{G}_{0}^{(3 / 2)}(p)=\frac{1}{\left(2 \cosh \frac{\pi p}{2}\right)^{2}}, \quad \hat{G}_{m}^{(3 / 2)}(p)=-\frac{1}{\left(2 \cosh \frac{\pi p}{2}\right)^{2}} e^{\pi p\left(\frac{2 m}{3}-1\right)} .
\end{array}
$$

We can numerically (by using Mathematica for $2 N=2,3, \cdots, 10$ ) prove the general formulæ, $1 \leq m \leq 2 N-1$ :

$$
\begin{array}{ll}
\hat{G}_{0}^{(N)}(p)=\frac{1}{\left(2 \cosh \frac{\pi p}{2}\right)^{2}} & \Rightarrow G_{0}^{(N)}(x)=\frac{1}{2 \pi^{2}} \frac{x}{\sinh x} \\
\hat{G}_{m}^{(N)}(p)=-\frac{1}{\left(2 \cosh \frac{\pi p}{2}\right)^{2}} e^{\pi p\left(\frac{m}{N}-1\right)} & \Rightarrow G_{m}^{(N)}(x)=\frac{1}{2 \pi^{2}} \frac{x-i \pi\left(\frac{m}{N}-1\right)}{\sinh \left(x-i \pi \frac{m}{N}\right)}
\end{array}
$$

Equations (3.19) with kernels (3.20) are valid in the strip defined by the conditions $\mid \operatorname{Im} \theta-$ $\phi_{n}+\phi_{m} \pm \epsilon \mid<\min \{\pi, \pi / N\}, \forall n, m$. They can be solved analytically in few cases, which we report in the next section, or alternatively by numerical iteration: we will discuss many examples in section 5 . We observe the simple summation

$$
\sum_{m=0}^{2 N-1} \hat{G}_{m}(p)=\frac{\sinh \left(\frac{\pi p}{2 N}-\frac{\pi p}{2}\right)}{2 \cosh \frac{\pi p}{2} \sinh \frac{\pi p}{2 N}}=\left.\hat{G}^{L Z}(p)\right|_{\alpha=N},
$$

where $\left.\hat{G}^{L Z}(p)\right|_{\alpha=N}$ is the Fourier transform of the kernel of equation (3.32) of [20] (and of (2.29) of [34] which gives hints on why and how wall-crossing changes the Fourier transforms according to the analyticity regions (5.9) and $(5.10)$ of $\left.[35]^{6}\right)$ to which our equations (3.19) reduce in the case in which $Z\left(\theta ; k, \vec{c}_{n}\right)$ do not depend on $n$.

Once we know (the unique) $Z$, we can extend the usual formula for the free energy [32, 33 ] and very reasonably conjecture that it be given by the functional ${ }^{7}$

$F=\sum_{n=0}^{2 N-1} \int_{-\infty}^{+\infty} \frac{d \theta}{2 \pi}\left[f_{n}(\theta+i \epsilon) L_{+}\left(\theta-i \phi_{n} ; k, \vec{c}_{n}\right)-f_{n}(\theta-i \epsilon) L_{-}\left(\theta-i \phi_{n} ; k, \vec{c}_{n}\right)\right]+(k \rightarrow-k)$,

\footnotetext{
${ }^{6}$ This new way to wall-crossing will be pursued in [16].

${ }^{7}$ Interestingly, the property $\bar{Z}(\theta ; k, \vec{c})=-Z(-\bar{\theta} ;-k, \vec{c})$, which descends from (2.23), is enough to ensure that the quantity given by $(3.22)$ is real.
} 
with $f_{n}(\theta)=\left|r\left(\vec{c}_{n}\right)\right| \sinh \theta$, so that (when $l=0$ ) it must coincide with the TBA contribution $[14,36]$

$$
A_{\text {free }}=\sum_{s=1}^{N_{\max }} \int_{-\infty}^{+\infty} \frac{d \theta}{2 \pi}\left|m_{s}\right| \cosh \theta \ln \left[1+\tilde{Y}_{s}^{A M S V}(\theta)\right],
$$

to the area (scattering amplitude) $A=A_{\text {free }}+A_{\text {periods }}$ of $4 N+4$ gluons. The functions $\tilde{Y}_{s}^{A M S V}(\theta)$, whose number $N_{\max }$ depends on the parameters $\vec{c}$ and varies from $2 N-1$ (minimal chamber) to $(2 N-1) N$ (maximal chamber), satisfy TBA equations. For parameters in minimal chamber TBA equations have the form (26) of [14]:

$$
\begin{aligned}
\ln \tilde{Y}_{s}^{A M S V}(\theta) & =-\left|m_{s}\right| \cosh \theta+\sum_{s^{\prime}=1}^{2 N-1} \int d \theta^{\prime} K_{s, s^{\prime}}\left(\theta-\theta^{\prime}\right) \ln \left(1+\tilde{Y}_{s^{\prime}}^{A M S V}\right)\left(\theta^{\prime}\right), \\
K_{s, s^{\prime}}(\theta) & =\frac{\delta_{s, s^{\prime}+1}+\delta_{s, s^{\prime}-1}}{2 \pi \cosh \left(\theta+i \varphi_{s}^{\mathrm{TBA}}-i \varphi_{s^{\prime}}^{\mathrm{TBA}}\right)}, \quad s=1, \ldots, 2 N-1 .
\end{aligned}
$$

Despite its simplicity and elegance, this coincidence is not easy to be proven analytically. Therefore, in next sections we test the conjectured identity in the lower cases where analytic or numerical computations can be performed and leave the analytical proof for future publication about the full-fledged connexion between the unique NLIE variable $Z$ and those of TBA, $Y$ s. Nevertheless, we anticipate here the relation between the first function $Y_{1}^{A M S V}(\theta)=\tilde{Y}_{1}^{A M S V}\left(\theta-i \varphi_{1}\right)$ and the counting function (which relation can easily be proven by expressing $Z$ and $Y$ s in terms of the $Q$-function [16]):

$$
1+Y_{1}^{A M S V}(\theta)=\left[1+e^{-i Z\left(\theta-\frac{i \pi}{2} ; \pm \frac{1}{4}, \vec{c}\right)}\right]\left[1+e^{i Z\left(\theta+\frac{i \pi}{2} ; \pm \frac{1}{4}, \vec{c}\right)}\right] .
$$

Then, we shall take into consideration the $Y$-system to construct the higher $Y$ s. Yet, in this way we do not produce nice expressions for the $Y \mathrm{~s}$, at least not so manageable to accomplish the proof of the aforementioned identity (of (3.22) and (3.23)). Moreover, to this aim, one should also find the suitable relation between $r\left(\vec{c}_{n}\right)$ in (3.22) and $m_{s}$ in (3.23), which is not immediate, as well, in view of their rather different definitions. ${ }^{8}$

Before ending this part, we want to stress that NLIEs (3.19) are valid if $\left|c_{n}\right|, n=$ $1, \ldots, 2 N-2$ and, consequently the phases $\phi_{n}$, are not too large: to be precise, remembering what we wrote just after equation (3.20), the condition $\left|\phi_{n}-\phi_{m}\right|<\min \{\pi, \pi / N\}, \forall n, m$ has to be satisfied. In this case, we have $2 N$ NLIEs in the form (3.19). For general $c_{n}$ the form of the NLIEs changes and we expect the occurrence of a phenomenon analogous to the "wall crossing' of TBA equations [14, 15, 36]: in the context of integrability this phenomenon already appeared and was called 'second determination' of the NLIEs [32, 33], but its understanding can benefit much from its view [15] in the ODE/IM context [16].

As we discussed in the introduction, the main importance of the NLIEs in the context of integrability is that one can express all the relevant functions of the problem in terms of the counting function(s). If needed, the connexion to the $Y$-functions descends from (3.25).

\footnotetext{
${ }^{8}$ This identification is a long-standing problem since the first example of NLIE for the quantum SineGordon (SG) theory [32, 33], always confirmed only by numerical checks. Actually, our relation (3.25) is enough to prove it for (infinite) special values of the SG coupling.
} 
For instance, the transfer matrix is a functional of the counting function. In the simplest case without dependence on the moduli, for the ground state eigenvalue we find, following procedures discussed in [34, 35],

$$
T(\theta)=e^{\frac{2 i \pi k}{1+N}} \exp [F(\theta ; k)]+e^{-\frac{2 i \pi k}{1+N}} \exp \left[-F\left(\theta-i \frac{\pi}{N} ; k\right)\right],
$$

where, when $0<\operatorname{Im} \theta<\pi$,

$$
F(\theta ; k)=r \tan \frac{\pi}{2 N} \cosh \theta-\int_{-\infty}^{+\infty} \frac{d \theta^{\prime}}{2 i \pi} \frac{1}{\sinh \left(\theta-\theta^{\prime}\right)} \ln \frac{1+e^{i Z\left(\theta^{\prime}+i \epsilon ; k\right)}}{1+e^{-i Z\left(\theta^{\prime}-i \epsilon ; k\right)}},
$$

and, when $-\pi / N<\operatorname{Im} \theta<0$,

$$
F(\theta ; k)=-i \frac{r}{\cos \frac{\pi}{2 N}} \cosh \left(\theta+\frac{i \pi}{2 N}\right)+2 \pi i k+\int_{-\infty}^{+\infty} \frac{d \theta^{\prime}}{2 i \pi} G_{T}\left(\theta-\theta^{\prime}\right) \ln \frac{1+e^{i Z\left(\theta^{\prime}+i \epsilon ; k\right)}}{1+e^{-i Z\left(\theta^{\prime}-i \epsilon ; k\right)}},
$$

with

$$
G_{T}(x)=\int_{-\infty}^{+\infty} d k e^{-i k x+\frac{\pi k}{2 N}} \frac{i \tanh \frac{\pi k}{2}}{\sinh \left(\frac{\pi k}{2}+\frac{\pi k}{N}\right)} .
$$

Finally, the ground state eigenvalue of the $Q$ function in the simplest case with no moduli has been written in formulæ (3.38)-(3.40) of [20] and therefore we refer to that paper for explicit expressions. Formulæ for the eigenvalues of the $T$ and $Q$ functions in terms of $Z$ in the general case with arbitrary moduli are only technical complications and therefore for the moment we do not write them.

In the next sections, we prove the identity analytically for the first cases and then numerically, as its full proof and analysis is outside the aim of this paper.

\section{Analytic computations}

Regular polygons. The regular polygon case corresponds to polynomials

$$
p(z)=z^{2 N} .
$$

This means that $c_{2 N}=1$, whilst all the other moduli $c_{n}=0, n=0,1, \ldots, 2 N-1$. This is the 'massless' limit $w_{0}(\vec{c})=0$ : in this limit, the evaluation of the free energy is done by using the 'dilogarithmic trick' applied to NLIEs (see [32, 33]). We have that

$$
F=2 N \frac{\pi}{6} c_{\text {eff }},
$$

where the effective central charge $c_{\text {eff }}$ is easily computed (following section 7.1 of the first of $[32,33])$ as

$$
c_{\mathrm{eff}}=1-\frac{3}{2(1+N)}=\frac{2 N-1}{2 N+2} .
$$

Then, from (4.3) and (4.2) we get the free energy

$$
F=\frac{\pi}{6} 2 N \frac{2 N-1}{2 N+2},
$$

which agrees with (4.7) of [36], since the number $n$ there is expressed by our $N$ by

$$
n=2 N+2 .
$$


The octagon. Another case in which explicit analytic computation is possible is the octagon: $N=1, p(z)=z^{2}+c_{0}$. This case corresponds in the ODE limit to the harmonic oscillator and the solutions of the two NLIEs are ${ }^{9}$

$$
Z(\theta ; k, \vec{c})=Z\left(\theta ; k, \vec{c}_{1}\right)=\pi\left|c_{0}\right| \sinh \theta-2 \pi k, \quad \vec{c}=\left(c_{0}, 0,1\right) .
$$

In order to evaluate the free energy it is useful to rewrite (3.22) as:

$$
F=\pi\left|c_{0}\right| \operatorname{Im} \int \frac{d \theta}{2 \pi} \sinh (\theta+i \epsilon) 2 \ln \left[1+e^{i \pi\left|c_{0}\right| \sinh (\theta+i \epsilon)-2 i \pi k}\right]+(k \rightarrow-k) .
$$

We stick to the case $l=0$, that is $k=1 / 4$. Then, we choose the arbitrary constant $\epsilon=\pi / 2$ : with these positions

$$
F=\pi\left|c_{0}\right| \operatorname{Im} \int \frac{d \theta}{2 \pi} i \cosh \theta 2 \ln \left[1+e^{-2 \pi\left|c_{0}\right| \cosh \theta}\right] .
$$

Expanding the logarithm in series, we find the result as a series of Bessel functions $K_{1}$ :

$$
F=-2\left|c_{0}\right| \sum_{n=1}^{+\infty} \frac{(-1)^{n}}{n} K_{1}\left(2 \pi\left|c_{0}\right| n\right),
$$

which agrees with computations made [37] using minimal area.

\section{Numerical computations}

We report some numerical computations of free energy. We test our NLIEs against the TBA equations in $[14,26]$. Let us first consider the polynomial $p(z, \vec{c})=z^{4}+c_{2} z^{2}+c_{1} z-1$, where $c_{1}$ and $c_{2}$ are chosen to keep the TBA equations in maximal chamber. In this case, the TBA equations are given by ${ }^{10}$

$$
\begin{aligned}
\widetilde{\epsilon}_{1} & =\left|m_{1}\right| \cosh \theta-K_{1,2} \star \widetilde{L}_{2}-K_{1,12}^{+} \star \widetilde{L}_{12}-K_{1,23}^{+} \star \widetilde{L}_{23}-K_{1,123}^{+} \star \widetilde{L}_{123}, \\
\widetilde{\epsilon}_{2} & =\left|m_{2}\right| \cosh \theta-K_{2,1} \star \widetilde{L}_{1}-K_{2,3} \star \widetilde{L}_{3}-K_{2,12} \star \widetilde{L}_{12}-K_{2,23} \star \widetilde{L}_{23}-2 K_{2,123} \star \widetilde{L}_{123}, \\
\widetilde{\epsilon}_{3} & =\left|m_{3}\right| \cosh \theta-K_{3,2} \star \widetilde{L}_{2}-K_{3,12}^{+} \star \widetilde{L}_{12}-K_{3,23}^{+} \star \widetilde{L}_{23}-K_{3,123}^{+} \star \widetilde{L}_{123}, \\
\widetilde{\epsilon}_{12} & =\left|m_{12}\right| \cosh \theta-K_{12,1}^{-} \star \widetilde{L}_{1}-K_{12,3}^{-} \star \widetilde{L}_{3}-K_{12,2} \star \widetilde{L}_{2}-K_{12,123}^{-} \star \widetilde{L}_{123}, \\
\widetilde{\epsilon}_{23} & =\left|m_{23}\right| \cosh \theta-K_{23,1}^{-} \star \widetilde{L}_{1}-K_{23,3}^{-} \star \widetilde{L}_{3}-K_{23,2} \star \widetilde{L}_{2}-K_{23,123}^{-} \star \widetilde{L}_{123}, \\
\widetilde{\epsilon}_{123} & =\left|m_{123}\right| \cosh \theta-K_{123,1}^{-} \star \widetilde{L}_{1}-K_{123,3}^{-} \star \widetilde{L}_{3}-2 K_{123,2} \star \widetilde{L}_{2}-K_{123,12}^{+} \star \widetilde{L}_{12}-K_{123,23}^{+} \star \widetilde{L}_{23} .
\end{aligned}
$$

where $\tilde{\epsilon}_{j}=-\ln \tilde{Y}_{j}^{A M S V}, \tilde{L}_{j}=\ln \left(1+e^{-\tilde{\epsilon}_{j}}\right)$ and $\star$ denotes the convolution. The kernels in the integral equations are

$$
K_{r, s}=\frac{1}{2 \pi} \frac{1}{\cosh \left(\theta+i\left(\operatorname{Arg}\left(m_{s}\right)-\operatorname{Arg}\left(m_{r}\right)\right)\right)} .
$$

\footnotetext{
${ }^{9}$ In this case the quantity $r_{0}$ which is constructed from the zero modes vanishes and the driving term is due only to the additional term $\delta r$.

${ }^{10}$ In our notation, the masses $m_{a}$ are twice of the masses in [26].
} 


\begin{tabular}{|c|c|c|}
\hline$c_{2}$ & $r(\vec{c})$ & $r\left(\vec{c}_{1}\right)$ \\
\hline 1 & $2.582636248747118^{\prime}$ & $5.049379803768095^{\prime}$ \\
\hline$\frac{1}{2}$ & $2.973818997047456^{\prime}$ & $4.181170379881933^{\prime}$ \\
\hline 0 & $3.4960767390561593^{\prime}$ & $3.4960767390561593^{\prime}$ \\
\hline
\end{tabular}

Table 1. $r(\vec{c})$ and $r\left(\vec{c}_{1}\right): p(z, \vec{c})=z^{4}+c_{2} z^{2}-1$. Other $r(\vec{c})$ are obtained by $r\left(\vec{c}_{2}\right)=r(c)$ and $r\left(\vec{c}_{3}\right)=r\left(\vec{c}_{1}\right)$.

\begin{tabular}{|c|c|c|c|}
\hline$c_{2}$ & $\frac{6}{\pi} F$ & $\frac{6}{\pi} A_{\text {free }}$ & $F / A_{\text {free }}$ \\
\hline 1 & $0.03710467901895455^{\prime}$ & $0.03710480745177178^{\prime}$ & $0.9999965386475216^{\prime}$ \\
\hline$\frac{1}{2}$ & $0.04910296244435445^{\prime}$ & $0.049102990179460966^{\prime}$ & $0.9999994351646119^{\prime}$ \\
\hline 0 & $0.055363520349671534^{\prime}$ & $0.0553655493634881^{\prime}$ & $0.9999633524124677^{\prime}$ \\
\hline
\end{tabular}

Table 2. Numeric check in maximal chamber: $p(z, \vec{c})=z^{4}+c_{2} z^{2}-1$. The NLIEs are solved by Fourier discretisation with cutoff $(-12,12)$ and $2^{12}$ points. The parameter $\epsilon$ in eq. (3.15) is fixed to be $1 / 2^{4}$. The TBA equations are solved by $2^{14}$ points and cutoff $(-20,20)$.

The superscripts of kernel denote the shift of the argument $f^{ \pm}(\theta)=f\left(\theta \pm \frac{\pi i}{2}\right)$. $A_{\text {free }}$ is now given by

$$
\begin{aligned}
A_{\text {free }}= & \frac{1}{2 \pi} \int_{-\infty}^{\infty}\left(\left|m_{1}\right| \cosh \theta \log \left(1+e^{-\tilde{\epsilon}_{1}}\right)+\left|m_{2}\right| \log \left(1+e^{-\tilde{\epsilon}_{2}}\right)+\left|m_{3}\right| \cosh \theta \log \left(1+e^{-\tilde{\epsilon}_{3}}\right)\right. \\
& \left.+\left|m_{12}\right| \log \left(1+e^{-\tilde{\epsilon}_{12}}\right)+\left|m_{23}\right| \log \left(1+e^{-\tilde{\epsilon}_{23}}\right)+\left|m_{123}\right| \log \left(1+e^{-\tilde{\epsilon}_{123}}\right)\right) d \theta .
\end{aligned}
$$

When $c_{1}=0$, we get the double well potential, where $m_{1}=m_{3}$ and $m_{12}=m_{23}$. The $r$ in NLIEs can be computed explicitly by using (3.8). In table 1, we list several examples for $r(\vec{c})$ and $r\left(\vec{c}_{1}\right)$. Other $r(\vec{c})$ are obtained by $r\left(\vec{c}_{2}\right)=r(\vec{c})$ and $r\left(\vec{c}_{3}\right)=r\left(\vec{c}_{1}\right)$. The free energy can be computed by solving the NLIEs (3.19) and then by substituting the numerical solution into equation (3.22) for the free energy. In table 2 we show the numerical results of $\frac{6}{\pi} F$ and $\frac{6}{\pi} A_{\text {free }}$ for several values of $c_{2}$. As expected, $F$ matches $A_{\text {free }}$ with a very high precision.

We then consider the polynomial with $c_{1} \neq 0$, for which the phase $\Phi(\theta, \vec{c})$ in equation (2.11) is nontrivial. Evaluating the masses (period integrals), we find $m_{1} \neq m_{3}$ and $m_{12} \neq m_{23}$. In table 3 and table 4 , we list $r_{0}(\vec{c})$ and $r(\vec{c})$, respectively, as defined in (3.8). In table 5 , we show the $\frac{6}{\pi} F$ and $\frac{6}{\pi} A_{\text {free }}$ for the polynomial $p(z, \vec{c})=z^{4}+c_{2} z-\frac{z}{200}-1$ with several $c_{2}$.

We then fix $c_{2}$ to be 1 , and vary $c_{1}$. In table 6 and table 7 , we list the $r_{0}(\vec{c})$ and $r(\vec{c})$ respectively for this case. In table 8 , we show the $\frac{6}{\pi} F$ and $\frac{6}{\pi} A_{\text {free }}$ for the polynomial $p(z, \vec{c})=z^{4}+z^{2}+c_{1} z-1$.

In a similar way, we have tested our NLIEs (3.15) for the polynomial $p(z, \vec{c})=z^{3}+$ $c_{1} z-1$ against the TBA equations in the maximal chamber. ${ }^{11}$ In table 9 , we present $\frac{6}{\pi} F$ and $\frac{6}{\pi} A_{\text {free }}$ for several values of $c_{1}$.

\footnotetext{
${ }^{11}$ The TBA equations can be found in equation (3.80) of [26].
} 


\begin{tabular}{|c|c|c|c|}
\hline$c_{2}$ & $r_{0}(\vec{c})$ & $r_{0}\left(\vec{c}_{1}\right)$ & $r_{0}\left(\vec{c}_{2}\right)$ \\
\hline 1 & $2.580322548331587^{\prime}$ & $5.049378533767665^{\prime}-0.002318241884034844^{\prime} i$ & $2.5849590320996567^{\prime}$ \\
\hline$\frac{1}{2}$ & $2.972598628551023^{\prime}$ & $4.181167930139899^{\prime}-0.0012249121993099799^{\prime} i$ & $2.9750484529496433^{\prime}$ \\
\hline$\frac{1}{100}$ & $3.4841068384904066^{\prime}$ & $3.5080872072271383^{\prime}-0.000024977324456942895^{\prime} i$ & $3.4841567931393205^{\prime}$ \\
\hline
\end{tabular}

Table 3. $r_{0}(\vec{c}), r_{0}\left(\vec{c}_{1}\right), r_{0}\left(\vec{c}_{2}\right)$ and $r_{0}\left(\vec{c}_{3}\right)$ for $p(z, \vec{c})=z^{4}+c_{2} z^{2}-\frac{z}{200}-1$. $r\left(\vec{c}_{3}\right)$ is obtained by $r_{0}\left(\vec{c}_{3}\right)=\bar{r}_{0}\left(\vec{c}_{1}\right)$.

\begin{tabular}{|c|c|c|c|}
\hline$c_{2}$ & $r(\vec{c})$ & $r\left(\vec{c}_{1}\right)$ & $r\left(\vec{c}_{2}\right)$ \\
\hline 1 & $2.5881765299655615^{\prime}$ & $5.049378533767665^{\prime}-0.010172223518009327^{\prime} i$ & $2.5771050504656823^{\prime}$ \\
\hline$\frac{1}{2}$ & $2.9804526101849973^{\prime}$ & $4.181167930139899^{\prime}-0.009078893833284463^{\prime} i$ & $2.967194471315669^{\prime}$ \\
\hline$\frac{1}{100}$ & $3.491960820124381^{\prime}$ & $3.5080872072271383^{\prime}-0.007878958958431426^{\prime} i$ & $3.476302811505346^{\prime}$ \\
\hline
\end{tabular}

Table 4. $r(\vec{c}), r\left(\vec{c}_{1}\right), r\left(\vec{c}_{2}\right)$ and $r\left(\vec{c}_{3}\right)$ for the polynomial $p(z, \vec{c})=z^{4}+c_{2} z^{2}-\frac{z}{200}-1$. $r\left(\vec{c}_{3}\right)$ is obtained by $r\left(\vec{c}_{3}\right)=\bar{r}\left(\vec{c}_{1}\right)$.

\begin{tabular}{|c|c|c|c|}
\hline$c_{2}$ & $\frac{6}{\pi} F$ & $\frac{6}{\pi} A_{\text {free }}$ & $F / A_{\text {free }}$ \\
\hline 1 & $0.037104841463217055^{\prime}$ & $0.03710484211496269^{\prime}$ & $0.9999999824350246^{\prime}$ \\
\hline$\frac{1}{2}$ & $0.04910375701712826^{\prime}$ & $0.04910376260115915^{\prime}$ & $0.9999998862809978^{\prime}$ \\
\hline$\frac{1}{100}$ & $0.05536470669758174^{\prime}$ & $0.055364704965062565^{\prime}$ & $1.0000000312928459^{\prime}$ \\
\hline
\end{tabular}

Table 5. Numeric check in maximal chamber: $p(z, \vec{c})=z^{4}+c_{2} z^{2}-\frac{z}{200}-1$. The NLIEs are solved by Fourier discretisation with cutoff $(-12,12)$ and $2^{12}$ points. The parameter $\epsilon$ in eq. (3.15) is fixed to be $1 / 2^{4}$. The TBA equations are solved by $2^{14}$ points and cutoff $(-20,20)$.

\begin{tabular}{|c|c|c|c|}
\hline$c_{1}$ & $r_{0}(\vec{c})$ & $r_{0}\left(\vec{c}_{1}\right)$ & $r_{0}\left(\vec{c}_{2}\right)$ \\
\hline$-\frac{1}{2}$ & $2.3933954392247605^{\prime}$ & $5.037001372706346^{\prime}-0.23424307835549674^{\prime} i$ & $2.8618815959357526^{\prime}$ \\
\hline$-\frac{1}{4}$ & $2.4777183763893467^{\prime}$ & $5.046201443952038^{\prime}-0.11622181204658544^{\prime} i$ & $2.7101620004825175^{\prime}$ \\
\hline$-\frac{1}{50}$ & $2.5734354362619722^{\prime}$ & $5.049359181799129^{\prime}-0.009273172229004922^{\prime} i$ & $2.591981780719982^{\prime}$ \\
\hline
\end{tabular}

Table 6. $r_{0}(\vec{c}), r_{0}\left(\vec{c}_{1}\right), r_{0}\left(\vec{c}_{2}\right)$ and $r_{0}\left(\vec{c}_{3}\right)$ for the polynomial $p(z, \vec{c})=z^{4}+z^{2}+c_{1} z-1$. $r_{0}\left(\vec{c}_{3}\right)$ is obtained by $r_{0}\left(\vec{c}_{3}\right)=\bar{r}_{0}\left(\vec{c}_{1}\right)$.

\begin{tabular}{|c|c|c|c|}
\hline$c_{1}$ & $r(\vec{c})$ & $r\left(\vec{c}_{1}\right)$ & $r\left(\vec{c}_{2}\right)$ \\
\hline$-\frac{1}{2}$ & $3.178793602622209^{\prime}$ & $5.037001372706346^{\prime}-1.019641241752945^{\prime} i$ & $2.0764834325383044^{\prime}$ \\
\hline$-\frac{1}{4}$ & $2.870417458088071^{\prime}$ & $5.046201443952038^{\prime}-0.5089208937453096^{\prime} i$ & $2.3174629187837934^{\prime}$ \\
\hline$-\frac{1}{50}$ & $2.60485136279787^{\prime}$ & $5.049359181799129^{\prime}-0.040689098764902856^{\prime} i$ & $2.5605658541840843^{\prime}$ \\
\hline
\end{tabular}

Table 7. $r(\vec{c}), r\left(\vec{c}_{1}\right), r\left(\vec{c}_{2}\right)$ and $r\left(\vec{c}_{3}\right)$ for the polynomial $p(z, \vec{c})=z^{4}+z^{2}+c_{1} z-1 . r\left(\vec{c}_{3}\right)$ is obtained by $r\left(\vec{c}_{3}\right)=\bar{r}\left(\vec{c}_{1}\right)$. 


\begin{tabular}{|c|c|c|c|}
\hline$c_{1}$ & $\frac{6}{\pi} F$ & $\frac{6}{\pi} A_{\text {free }}$ & $F / A_{\text {free }}$ \\
\hline$-\frac{1}{2}$ & $0.03704085160351035^{\prime}$ & $0.03704085160351035^{\prime}$ & 1.000000000000000 \\
\hline$-\frac{1}{4}$ & $0.03716372850655718^{\prime}$ & $0.03716372633241627^{\prime}$ & $1.000000058501693^{\prime}$ \\
\hline$-\frac{1}{50}$ & $0.03710536684451506^{\prime}$ & $0.037105361745906645^{\prime}$ & $1.0000001374089398^{\prime}$ \\
\hline
\end{tabular}

Table 8. Numeric check in maximal chamber: $p(z, \vec{c})=z^{4}+z^{2}+c_{1} z-1$. The NLIEs are solved by Fourier discretisation with cutoff $(-12,12)$ and $2^{10}$ points. The parameter $\epsilon$ in eq. (3.15) is fixed to be $1 / 2$. The TBA equations are solved by $2^{14}$ points and cutoff $(-20,20)$.

\begin{tabular}{|c|c|c|c|}
\hline$c_{1}$ & $\frac{6}{\pi} F$ & $\frac{6}{\pi} A_{\text {free }}$ & $F / A_{\text {free }}$ \\
\hline$-\frac{1}{2}$ & $0.027577390204736915^{\prime}$ & $0.027585003289666222^{\prime}$ & $0.9997240136298206^{\prime}$ \\
\hline$-\frac{1}{10}$ & $0.017482596263586746^{\prime}$ & $0.017482865359751576^{\prime}$ & $0.9999846080056505^{\prime}$ \\
\hline$-\frac{1}{100}$ & $0.01719846067124951^{\prime}$ & $0.017198793671935574^{\prime}$ & $0.9999806381370412^{\prime}$ \\
\hline
\end{tabular}

Table 9. $\frac{6}{\pi} F$ and $\frac{6}{\pi} A_{\text {free }}$ for $p(z, \vec{c})=z^{3}+c_{1} z-1$. The NLIEs are solved by Fourier discretization with cutoff $(-12,12)$ and $2^{12}$ points. The parameter $\epsilon$ in eq. $(3.15)$ is fixed to be $1 / 2^{4}$. The TBA equations are solved by $2^{14}$ points and cutoff $(-20,20)$.

Our numerical results show that

$$
F=A_{\text {free }},
$$

at least in the maximal chamber. In other chambers, we need to consider the wall crossing of NLIEs and TBA equations, which will be pursued in a future publication. At the end of section, it is worth to note that our NLIEs are much simpler than the TBA equations, especially when the number of cusps of Wilson loop is large. For the lightlike Wilson loop with $4 N+4$ cusps, one needs $2 N(2 N-1) / 2$ TBA equations to compute the free energy in maximal chamber, while the number of NLIEs is only $2 N$. Moreover, one needs to perform $(N-1)(2 N-1)$ times wall crossing to derive the TBA equations in the maximal chamber, which is quite complicated. Therefore, it is much convenient to use the NLIEs instead of TBA equation, especially in maximal chamber.

\section{Conformal limit}

Consider the NLIEs in the limit

$$
\left|c_{0}\right| \rightarrow 0, \quad c_{n}=\left|c_{0}\right|^{\frac{2 N-n}{2 N}} c_{n}^{c} \rightarrow 0, \quad \theta=\theta^{c}-\frac{1+N}{2 N} \ln \left|c_{0}\right| \rightarrow+\infty,
$$

with $c_{n}^{c}, \theta^{c}$ finite. Then, we have that

$$
w_{0}(\vec{c}) e^{\theta} \rightarrow w_{0}\left(\vec{c}^{c}\right) e^{\theta^{c}}, \quad \bar{w}_{0}(\vec{c}) e^{-\theta} \rightarrow 0, \quad B_{-1}(\vec{c}) e^{\theta} \rightarrow B_{-1}\left(\vec{c}^{c}\right) e^{\theta^{c}}, \quad \bar{B}_{-1}(\vec{c}) e^{-\theta} \rightarrow 0 .
$$

The linear problem in the variable $z$, after the scaling

$$
z=x e^{-\frac{\theta}{1+N}},
$$




\begin{tabular}{|c|c|c|c|}
\hline$c_{1}^{c}$ & $r_{0}\left(\vec{c}^{c}\right)$ & $r_{0}\left(\vec{c}_{1}^{c}\right)$ & $r_{0}\left(\vec{c}_{2}^{c}\right)$ \\
\hline$-\frac{1}{100}$ & $3.4960916735302283^{\prime}$ & $3.4960617618285434^{\prime}$ & $3.496091757138591^{\prime}$ \\
\hline$-\frac{1}{10}$ & $3.4975335418072313^{\prime}$ & $3.4945799178619184^{\prime}-0.00004172438919569643^{\prime} i$ & $3.4976169905856227^{\prime}$ \\
\hline
\end{tabular}

Table 10. $r_{0}\left(\vec{c}_{n}^{c}\right)$ in the conformal limit. $r_{0}\left(\vec{c}_{3}^{c}\right)$ is obtained by conjugating $r_{0}\left(\vec{c}_{1}^{c}\right)$.

becomes

$$
\left[-\frac{d^{2}}{d x^{2}}+\frac{l(l+1)}{x^{2}}+\sum_{n=1}^{2 N} c_{n}^{c} e^{\frac{2 N-n}{1+N} \theta^{c}} x^{n}\right] \psi(x)=e^{\frac{2 N}{1+N} \theta^{c}} \psi(x)
$$

which is a Schrödinger problem with energy $E=e^{\frac{2 N}{1+N} \theta^{c}}$ and potential

$$
V(x)=\sum_{n=1}^{2 N} c_{n}^{c} e^{\frac{2 N-n}{1+N} \theta^{c}} x^{n}
$$

Interestingly, the limiting $\hat{\Omega}$ symmetry acts as

$$
\hat{\Omega}_{\lim }: \quad x \rightarrow x e^{\frac{i \pi}{1+N}}, \quad c_{n}^{c} e^{\frac{2 N-n}{1+N} \theta^{c}} \rightarrow c_{n}^{c} e^{\frac{2 N-n}{1+N} \theta^{c}} e^{i \pi \frac{2 N-n}{1+N}}, \quad e^{\frac{2 N}{1+N} \theta^{c}} \rightarrow e^{\frac{2 N}{1+N} \theta^{c}} e^{-\frac{2 i \pi}{1+N}}
$$

and the limiting $\hat{\Pi}$ symmetry as

$$
\hat{\Pi}_{\lim }: \quad x \rightarrow x e^{-\frac{i \pi}{1+N}}, \quad c_{n}^{c} e^{\frac{2 N-n}{1+N} \theta^{c}} \rightarrow c_{n}^{c} e^{\frac{2 N-n}{1+N} \theta^{c}} e^{-i \pi \frac{2 N-n}{1+N}}, \quad e^{\frac{2 N}{1+N} \theta^{c}} \rightarrow e^{\frac{2 N}{1+N} \theta^{c}} e^{\frac{2 i \pi}{1+N}} .
$$

Therefore in the ODE limit $\hat{\Omega}_{\lim }=\left(\hat{\Pi}_{\lim }\right)^{-1}$ and then one can use only one type of symmetry.

In this limit, we make of course contact with known results. For instance, if $p(z, \vec{c})=$ $z^{2 N}+c_{N-1} z^{N-1}+c_{0}$, then $B_{-1}(\vec{c})=c_{N-1} / 2$ and the extra driving term of the NLIEs, $-\frac{\pi}{N} B_{-1}\left(\vec{c}^{c}\right) e^{\theta^{c}}=\frac{-\pi c_{N-1}^{c f t}}{2 N} e^{\theta^{c}}$, equals $-\pi / 2 N$ times the coefficient of the term $x^{N-1}$ in $V(x)$, in agreement with [17] and [18].

To complete our comparison between our NLIEs in conformal limit and the NLIEs found in [17] and [18], we report here some numerical results. We consider as an example the polynomial $p(z, \vec{c})=z^{4}+\left|c_{0}\right|^{3 / 4} c_{1}^{c} z-\left|c_{0}\right|$ with a small negative $c_{0}$. Under the limit (6.1), $w_{0}(\vec{c}) e^{\theta}$ becomes

$$
w_{0}(\vec{c}) e^{\theta} \rightarrow-\int_{0}^{\infty} d \tilde{z}\left[\sqrt{\tilde{z}^{4}+e^{\frac{3 \pi i}{4}} c_{1}^{c} \tilde{z}+1}-q_{2}(\tilde{z})\right] e^{\theta^{c}}
$$

where $z=\left|c_{0}\right|^{1 / 4} \tilde{z}$ and $q_{2}(\tilde{z})$ is the related polynomial which makes the integration convergent. In table 10 and 11, we list $r_{0}\left(\vec{c}^{c}\right)$ and $r\left(\vec{c}^{c}\right)$. Moreover, the driving terms in NLIEs (3.19) become $\frac{1}{2}\left|r\left(\vec{c}_{n}^{c}\right)\right| e^{\theta^{c}}-2 \pi k$ in the conformal limit, while the rest of the equations stays the same in form. With this information we evaluate (3.19) in the conformal limit numerically. 


\begin{tabular}{|c|c|c|c|}
\hline$c_{1}^{c}$ & $r\left(\vec{c}^{c}\right)$ & $r\left(\vec{c}_{1}^{c}\right)$ & $r\left(\vec{c}_{2}^{c}\right)$ \\
\hline$-\frac{1}{100}$ & $3.511799636798177^{\prime}$ & $3.4960617618285434^{\prime}-0.015707963267948967^{\prime} i$ & $3.4803837938706423^{\prime}$ \\
\hline$-\frac{1}{10}$ & $3.654613174486721^{\prime}$ & $3.4945799178619184^{\prime}-0.15712135706868535^{\prime} i$ & $3.340537357906133^{\prime}$ \\
\hline
\end{tabular}

Table 11. $r\left(\vec{c}_{n}^{c}\right)$ in the conformal limit. $r\left(\vec{c}_{3}^{c}\right)$ is obtained by conjugating $r\left(\vec{c}_{1}^{c}\right)$.

On the other hand, starting from $p(z)=z^{4}+\left|c_{0}\right|^{3 / 4} c_{1}^{c} z-\left|c_{0}\right|$, the potential (6.5) becomes $V(x)=x^{4}+c_{1}^{c} e^{\theta^{c}} x$, from which one finds the NLIEs [17] and [18]:

$$
\begin{aligned}
\ln a_{ \pm}\left(\theta^{c}\right)= & \frac{\pi i}{2}\left(2 l+1 \pm \frac{\alpha}{2}\right)-i b_{0} e^{\theta^{c}} \\
& +\int_{\mathcal{C}_{1}} d \theta^{\prime} K_{1}\left(\theta^{c}-\theta^{\prime}\right) \ln \left(1+a_{ \pm}\left(\theta^{\prime}\right)\right)-\int_{\mathcal{C}_{2}} d \theta^{\prime} K_{1}\left(\theta^{c}-\theta^{\prime}\right) \ln \left(1+\frac{1}{a_{ \pm}\left(\theta^{\prime}\right)}\right) \\
& +\int_{\mathcal{C}_{1}} d \theta^{\prime} K_{2}\left(\theta^{c}-\theta^{\prime}\right) \ln \left(1+a_{\mp}\left(\theta^{\prime}\right)\right)-\int_{\mathcal{C}_{2}} d \theta^{\prime} K_{2}\left(\theta^{c}-\theta^{\prime}\right) \ln \left(1+\frac{1}{a_{\mp}\left(\theta^{\prime}\right)}\right)
\end{aligned}
$$

where $\alpha=c_{1}^{c} e^{\theta^{c}}$ and the kernels $K_{1}$ and $K_{2}$ are given by

$$
\begin{aligned}
& K_{1}\left(\theta^{c}\right)=-\left(\frac{1}{4 \pi \cosh \theta^{c}}-\frac{\theta^{c}}{2 \pi^{2} \cosh \theta^{c} \sinh \theta^{c}}\right), \\
& K_{2}\left(\theta^{c}\right)=-\left(\frac{1}{4 \pi \cosh \theta^{c}}+\frac{\theta^{c}}{2 \pi^{2} \cosh \theta^{c} \sinh \theta^{c}}\right) .
\end{aligned}
$$

$\mathcal{C}_{1}$ and $\mathcal{C}_{2}$ are integration contours just below and just above the real $\theta^{c}$ axis with a distance $\epsilon$. We introduce the related free energy obtained from these NLIEs by

$$
\begin{aligned}
\mathcal{F}= & \frac{i}{\pi} \int d \theta^{c}\left(\left(b_{0}+\frac{\pi}{4} c_{1}^{c}\right) e^{\theta^{c}-i \epsilon} \ln \left(1+a_{+}\left(\theta^{c}-i \epsilon\right)\right)+\left(b_{0}-\frac{\pi}{4} c_{1}^{c}\right) e^{\theta^{c}-i \epsilon} \ln \left(1+a_{-}\left(\theta^{c}-i \epsilon\right)\right)\right. \\
& \left.-\left(b_{0}+\frac{\pi}{4} c_{1}^{c}\right) e^{\theta^{c}+i \epsilon} \ln \left(1+\frac{1}{a_{+}\left(\theta^{c}+i \epsilon\right)}\right)-\left(b_{0}-\frac{\pi}{4} c_{1}^{c}\right) e^{\theta^{c}+i \epsilon} \ln \left(1+\frac{1}{a_{-}\left(\theta^{c}+i \epsilon\right)}\right)\right) .
\end{aligned}
$$

Then, our free energy in the conformal limit $F^{c}$ and $\mathcal{F}$ lead to the same result

$$
F^{c}=\frac{\pi}{6}=\mathcal{F},
$$

when $l=0$. In order to understand the precise relationship between our NLIEs and the ones of (6.9), we solve both numerically. We evaluate the two type of NLIEs by Fourier discretization with a cutoff $(-12,12)$ and $2^{10}$ points. The parameter $\epsilon$ related with integration contour is fixed to be $1 / 2^{4}$. In figure 2 , we plot the real and imaginary parts of the functions $-i Z\left(\theta^{c}-i \phi_{n}, \vec{c}_{n}^{c}\right)$ and $\ln a_{ \pm}\left(\theta^{c}\right)$, respectively, with fixed $c_{1}^{c}=-\frac{1}{100}$ and $l=0$. In figure 3 , we plot the real and imaginary parts of the functions $-i Z\left(\theta^{c}-i \phi_{n}, \vec{c}_{n}^{c}\right)$ and $\ln a_{ \pm}\left(\theta^{c}\right)$, respectively, with fixed $c_{1}^{c}=-\frac{1}{10}$ and $l=0$. In larger scale of $\theta$, we also find the similar behavior.

These numeric results (and many other examples) suggest

$$
-i Z\left(\theta^{c}-i \phi_{0}, \vec{c}^{c}\right)=\ln a_{+}\left(\theta^{c}\right), \quad-i Z\left(\theta^{c}-i \phi_{2}, \vec{c}_{2}^{c}\right)=\ln a_{-}(\theta) .
$$



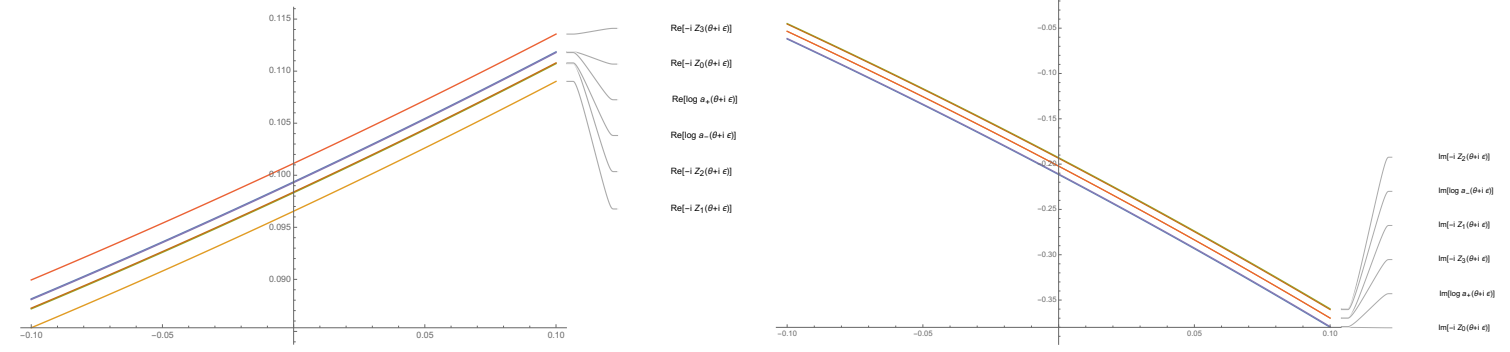

Figure 2. The real (left) and imaginary (right) parts (right) of the functions $-i Z\left(\theta^{c}-i \phi_{n}, \vec{c}_{n}^{c}\right)$ and $\ln a_{ \pm}\left(\theta^{c}\right)$ with $c_{1}^{c}=-\frac{1}{100}$.
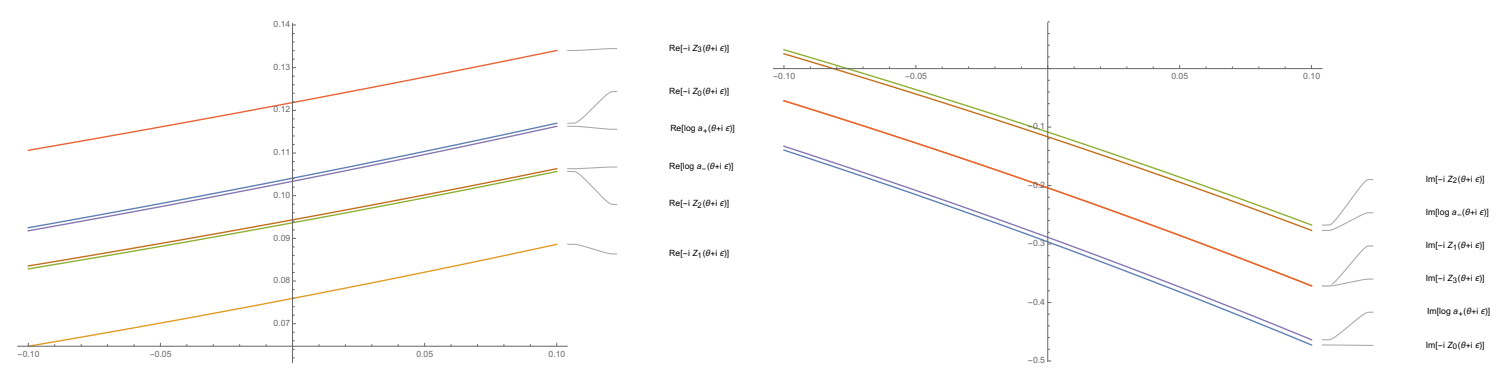

Figure 3. The real (left) and imaginary (right) parts of the functions $-i Z\left(\theta^{c}-i \phi_{n}, \vec{c}_{n}^{c}\right)$ and $\ln a_{ \pm}\left(\theta^{c}\right)$ with $c_{1}^{c}=-\frac{1}{10}$ and $l=0$.

From the numeric solution, we also find

$$
\begin{aligned}
Z\left(\theta^{c}-i \phi_{0}, \vec{c}^{c}\right)+Z\left(\theta^{c}-i \phi_{2}, \vec{c}_{2}^{c}\right) & =Z\left(\theta-i \phi_{1}, \vec{c}_{1}^{c}\right)+Z\left(\theta-i \phi_{3}, \vec{c}_{3}^{c}\right) \\
\operatorname{Re}\left(Z\left(\theta^{c}-i \phi_{1}, \vec{c}_{1}^{c}\right)\right) & =\operatorname{Re}\left(Z\left(\theta^{c}-i \phi_{3}, \vec{c}_{3}^{c}\right)\right)
\end{aligned}
$$

Moreover, we also find numerically the same relations (6.13) and (6.14) for various values of $l$ between $-\frac{1}{2}$ and $+\frac{1}{2}$. Then, we can very reasonably conclude that the conformal limit of our NLIEs (3.19) reproduce the results of [17] and [18].

\section{Conclusions}

We have constructed the main functional relations, $Q Q$ - and $T Q$-systems, underlying the (integrable) computations of the gluon scattering amplitudes in (planar) $\mathcal{N}=4 \mathrm{SYM}$ at strong coupling. Kinematical data of scattered gluons are encapsulated in complex quantities, the moduli, which appear 'dynamically' in the functional relations. In addition, we have provided a system of coupled NLIEs which renders effective the analytical and numerical explicit computation of these amplitudes. We tested our equations in the case of regular polygons (conformal limit) and the octagon ('harmonic oscillator' limit) by means of explicit analytic computations. In addition we provided various numerical tests for polynomials corresponding to polygons with ten and twelve sides. Possible applications of our functional and integral equations to the understanding of the BPS spectra and wall-crossing in $\mathcal{N}=2$ SYM [15] could become a very important issue on the desk. 
For the future, the principal aim is that our disentanglement of the integrability structure of the problem could shed light on the string quantisation, which, in the end, should reduce to a suitable deformation of the classical model (2.5), (2.1), (2.2), (2.3) or the derived functional equations or the Bethe Ansatz equations (3.5).

Then, it would be of interest to connect the linear problems with the strong coupling limit of an OPE series [38], as realised first in [39] for $A d S_{5}$ and then for all polygons in [40], eventually as their restriction to $A d S_{3}$ (with the fermionic bound state subtleties of [41]). In the opposite direction, it is very useful to generalise our method, based on $\mathrm{SU}(2)$, to the full $A d S_{5}$ (at strong coupling), the $\mathrm{SU}(4)$ Hitchin system indeed (for some generalisation to $\mathrm{SU}(3)$ cf. [42]).

In addition, the formalism discussed in this paper could be applied also to Wilson loops in Euclidean $A d S_{3}$, which problem becomes, after Pohlmeyer reduction, the modified cosh-Gordon equation [43].

An important final remark concerns the solution of classical equation (2.5). In a forthcoming publication [16] we show that it is obtained as a solution of an equation with the form of Gelfand-Levitan-Marchenko [44, 45] for which the inhomogeneous term (scattering data) is provided by $T$, the eigenvalue of the transfer matrix. This form of the equation is particularly important as it admits the solution to be written in terms of Fredholm determinants (i.e. tau functions)

$$
\eta=\hat{\eta}+\frac{1}{4} \ln P \bar{P}, \quad \hat{\eta}=\ln \frac{\operatorname{det}(1+K)}{\operatorname{det}(1-K)}
$$

and then, for instance, as a series expansion

$$
\hat{\eta}=\sum_{n=1}^{\infty} \hat{\eta}_{2 n-1}, \quad \hat{\eta}_{2 n-1}=\frac{2}{2 n-1} \int \prod_{i=1}^{2 n-1} \frac{d \theta_{i}}{4 \pi} T\left(\theta_{i}+\frac{i \pi}{2}+\frac{i \pi}{2 N}, \vec{c}\right) \frac{e^{-2 w e^{\theta_{i}}-2 \bar{w} e^{-\theta_{i}}}}{\cosh \frac{\theta_{i}-\theta_{i+1}}{2}} .
$$

$\eta$ is real due to the property $\bar{T}(\theta, \vec{c})=T(-\bar{\theta}, \vec{c})$, which comes from $(2.23),(2.26)$. And this is in fact the explicit solution of the initial problem, for $l=0$ the string problem, ${ }^{12}$ and we may think that a similar solution should exist for the more general $A d S_{5}$ case in terms of classical tau functions for the SU(4) Hitchin system.

\section{Acknowledgments}

D.F. thanks R. Tateo and D. Masoero for stimulating discussions. This work has been partially supported by the grants: GAST (INFN), the EC Network Gatis and the MIUR-PRIN contract 2017CC72MK_003. The work of H.S. has been supported by the grant "Exact Results in Gauge and String Theories" from the Knut and Alice Wallenberg foundation. H.S. would like to thank INFN and University of Bologna for the warm hospitality.

Open Access. This article is distributed under the terms of the Creative Commons Attribution License (CC-BY 4.0), which permits any use, distribution and reproduction in any medium, provided the original author(s) and source are credited.

\footnotetext{
${ }^{12}$ In fact, from it one can reconstruct the classical string world-sheet in $A d S_{3}$ by solving the two auxiliary linear problems at specific values of $\theta$.
} 


\section{References}

[1] J.M. Maldacena, The Large $N$ limit of superconformal field theories and supergravity, Int. J. Theor. Phys. 38 (1999) 1113 [Adv. Theor. Math. Phys. 2 (1998) 231] [hep-th/9711200] [INSPIRE].

[2] S.S. Gubser, I.R. Klebanov and A.M. Polyakov, Gauge theory correlators from noncritical string theory, Phys. Lett. B 428 (1998) 105 [hep-th/9802109] [INSPIRE].

[3] E. Witten, Anti-de Sitter space and holography, Adv. Theor. Math. Phys. 2 (1998) 253 [hep-th/9802150] [INSPIRE].

[4] N. Beisert et al., Review of AdS/CFT Integrability: An Overview, Lett. Math. Phys. 99 (2012) 3 [arXiv: 1012.3982] [INSPIRE].

[5] D. Bombardelli, D. Fioravanti and R. Tateo, Thermodynamic Bethe Ansatz for planar AdS/CFT: A Proposal, J. Phys. A 42 (2009) 375401 [arXiv:0902.3930] [InSPIRE].

[6] N. Gromov, V. Kazakov, A. Kozak and P. Vieira, Exact Spectrum of Anomalous Dimensions of Planar $N=4$ Supersymmetric Yang-Mills Theory: TBA and excited states, Lett. Math. Phys. 91 (2010) 265 [arXiv:0902.4458] [INSPIRE].

[7] G. Arutyunov and S. Frolov, Thermodynamic Bethe Ansatz for the AdS ${ }_{5} \times S^{5}$ Mirror Model, JHEP 05 (2009) 068 [arXiv:0903.0141] [INSPIRE].

[8] L.F. Alday and J.M. Maldacena, Gluon scattering amplitudes at strong coupling, JHEP 06 (2007) 064 [arXiv:0705.0303] [INSPIRE].

[9] P. Dorey and R. Tateo, Anharmonic oscillators, the thermodynamic Bethe ansatz, and nonlinear integral equations, J. Phys. A 32 (1999) L419 [hep-th/9812211] [INSPIRE].

[10] P. Dorey and R. Tateo, On the relation between Stokes multipliers and the T-Q systems of conformal field theory, Nucl. Phys. B 563 (1999) 573 [Erratum ibid. 603 (2001) 581] [hep-th/9906219] [INSPIRE].

[11] V.V. Bazhanov, S.L. Lukyanov and A.B. Zamolodchikov, Spectral determinants for Schrödinger equation and $Q$ operators of conformal field theory, J. Stat. Phys. 102 (2001) 567 [hep-th/9812247] [INSPIRE].

[12] V.V. Bazhanov, S.L. Lukyanov and A.B. Zamolodchikov, Higher level eigenvalues of $Q$ operators and Schroedinger equation, Adv. Theor. Math. Phys. 7 (2003) 711 [hep-th/0307108] [INSPIRE].

[13] D. Fioravanti, Geometrical loci and CFTs via the Virasoro symmetry of the mKdV-SG hierarchy: An Excursus, Phys. Lett. B 609 (2005) 173 [hep-th/0408079] [INSPIRE].

[14] L.F. Alday, J.M. Maldacena, A. Sever and P. Vieira, Y-system for Scattering Amplitudes, J. Phys. A 43 (2010) 485401 [arXiv:1002.2459] [InSPIRE].

[15] D. Gaiotto, G.W. Moore and A. Neitzke, Wall-crossing, Hitchin Systems, and the WKB Approximation, arXiv:0907.3987 [INSPIRE].

[16] D. Fioravanti and M. Rossi, ODE/IM correspondence, inverse scattering and Fredholm theory, to appear.

[17] J. Suzuki, Functional relations in Stokes multipliers: Fun with $x^{6}+\alpha x^{2}$ potential, J. Stat. Phys. 102 (2001) 1029 [quant-ph/0003066] [INSPIRE]. 
[18] P. Dorey, C. Dunning and R. Tateo, Spectral equivalences, Bethe Ansatz equations, and reality properties in PT-symmetric quantum mechanics, J. Phys. A 34 (2001) 5679 [hep-th/0103051] [INSPIRE].

[19] D. Fioravanti, F. Ravanini and M. Stanishkov, Generalized KdV and quantum inverse scattering description of conformal minimal models, Phys. Lett. B 367 (1996) 113 [hep-th/9510047] [INSPIRE].

[20] S.L. Lukyanov and A.B. Zamolodchikov, Quantum sine(h)-Gordon model and classical integrable equations, JHEP 07 (2010) 008 [arXiv:1003.5333] [INSPIRE].

[21] L.F. Alday and J.M. Maldacena, Minimal surfaces in AdS and the eight-gluon scattering amplitude at strong coupling, arXiv:0903.4707 [INSPIRE].

[22] J.M. Maldacena and A. Zhiboedov, Form factors at strong coupling via a Y-system, JHEP 11 (2010) 104 [arXiv: 1009.1139] [INSPIRE].

[23] C.R. Fernandez-Pousa, M.V. Gallas, T.J. Hollowood and J.L. Miramontes, The Symmetric space and homogeneous sine-Gordon theories, Nucl. Phys. B 484 (1997) 609 [hep-th/9606032] [INSPIRE].

[24] C.R. Fernandez-Pousa, M.V. Gallas, T.J. Hollowood and J.L. Miramontes, Solitonic integrable perturbations of parafermionic theories, Nucl. Phys. B 499 (1997) 673 [hep-th/9701109] [INSPIRE].

[25] J. Suzuki, Anharmonic oscillators, spectral determinant and short exact sequence of $\mathrm{U}_{q}\left(\widehat{\mathfrak{s}}_{2}\right)$, J. Phys. A 32 (1999) L183 [hep-th/9902053] [INSPIRE].

[26] K. Ito, M. Mariño and H. Shu, TBA equations and resurgent Quantum Mechanics, JHEP 01 (2019) 228 [arXiv: 1811.04812] [INSPIRE].

[27] K. Ito and H. Shu, TBA equations for the Schrödinger equation with a regular singularity, arXiv:1910.09406 [INSPIRE].

[28] D. Masoero, Y-System and Deformed Thermodynamic Bethe Ansatz, Lett. Math. Phys. 94 (2010) 151 [arXiv: 1005.1046] [INSPIRE].

[29] N. Gromov, V. Kazakov, S. Leurent and D. Volin, Quantum Spectral Curve for Planar $\mathcal{N}=4$ Super-Yang-Mills Theory, Phys. Rev. Lett. 112 (2014) 011602 [arXiv:1305.1939] [INSPIRE].

[30] A. Cavaglià, D. Fioravanti, N. Gromov and R. Tateo, Quantum Spectral Curve of the $\mathcal{N}=6$ Supersymmetric Chern-Simons Theory, Phys. Rev. Lett. 113 (2014) 021601 [arXiv: 1403.1859] [INSPIRE].

[31] D. Fioravanti and D. Gregori, Integrability and cycles of deformed $\mathcal{N}=2$ gauge theory, Phys. Lett. B 804 (2020) 135376 [arXiv: 1908.08030] [INSPIRE].

[32] C. Destri and H.J. De Vega, Unified approach to thermodynamic Bethe Ansatz and finite size corrections for lattice models and field theories, Nucl. Phys. B 438 (1995) 413 [hep-th/9407117] [INSPIRE].

[33] D. Fioravanti, A. Mariottini, E. Quattrini and F. Ravanini, Excited state Destri-De Vega equation for sine-Gordon and restricted sine-Gordon models, Phys. Lett. B 390 (1997) 243 [hep-th/9608091] [INSPIRE].

[34] D. Fioravanti and M. Rossi, Exact conserved quantities on the cylinder. 2. Off critical case, JHEP 08 (2003) 042 [hep-th/0302220] [INSPIRE]. 
[35] D. Fioravanti and M. Rossi, Exact conserved quantities on the cylinder 1: Conformal case, JHEP 07 (2003) 031 [hep-th/0211094] [INSPIRE].

[36] Y. Hatsuda, K. Ito, K. Sakai and Y. Satoh, Thermodynamic Bethe Ansatz Equations for Minimal Surfaces in $A d S_{3}$, JHEP 04 (2010) 108 [arXiv:1002.2941] [INSPIRE].

[37] L.F. Alday and J.M. Maldacena, Null polygonal Wilson loops and minimal surfaces in Anti-de-Sitter space, JHEP 11 (2009) 082 [arXiv:0904.0663] [INSPIRE].

[38] B. Basso, A. Sever and P. Vieira, Spacetime and Flux Tube S-Matrices at Finite Coupling for $N=4$ Supersymmetric Yang-Mills Theory, Phys. Rev. Lett. 111 (2013) 091602 [arXiv: 1303.1396] [INSPIRE].

[39] D. Fioravanti, S. Piscaglia and M. Rossi, Asymptotic Bethe Ansatz on the GKP vacuum as a defect spin chain: scattering, particles and minimal area Wilson loops, Nucl. Phys. B $\mathbf{8 9 8}$ (2015) 301 [arXiv: 1503.08795] [INSPIRE].

[40] A. Bonini, D. Fioravanti, S. Piscaglia and M. Rossi, Strong Wilson polygons from the lodge of free and bound mesons, JHEP 04 (2016) 029 [arXiv: 1511.05851] [INSPIRE].

[41] A. Bonini, D. Fioravanti, S. Piscaglia and M. Rossi, Fermions and scalars in $\mathcal{N}=4$ Wilson loops at strong coupling and beyond, Nucl. Phys. B 944 (2019) 114644 [arXiv:1807.09743] [INSPIRE].

[42] D. Fioravanti, H. Poghosyan and R. Poghossian, $T, Q$ and periods in $\mathrm{SU}(3) \mathcal{N}=2 S Y M$, JHEP 03 (2020) 049 [arXiv:1909.11100] [INSPIRE].

[43] M. Kruczenski, Wilson loops and minimal area surfaces in hyperbolic space, JHEP 11 (2014) 065 [arXiv: 1406.4945] [INSPIRE].

[44] I.M. Gelfand and B.M. Levitan, On the determination of a differential equation from its spectral function, in American Mathematical Society Translations 2, American Mathematical Society (1955), pp. 253-304 [Izv. Akad. Nauk SSSR Ser. Mat. 15 (1951) 309]

[45] V.A. Marchenko, Reconstruction of the potential energy from the phases of scattered waves, Dokl. Akad. Nauk SSSR 104 (1955) 433. 